OPEN ACCESS

Edited by: Abhay Satoskar,

The Ohio State University, United States

Reviewed by: Dominik Rückerl, The University of Manchester, United Kingdom

Fariborz Bahrami,

Pasteur Institute of Iran, Iran

*Correspondence: Grace Mulcahy grace.mulcahy@ucd.ie

Specialty section: This article was submitted to

Parasite Immunology,

a section of the journal

Frontiers in Immunology

Received: 22 June 2021

Accepted: 18 August 2021 Published: 20 September 2021

Citation:

Niedziela DA, Naranjo-Lucena $A$, Molina-Hernández V, Browne JA,

Martínez-Moreno Á, Pérez J, MacHugh DE and Mulcahy G (2021) Timing of Transcriptomic Peripheral Blood Mononuclear Cell Responses of

Sheep to Fasciola hepatica Infection Differs From Those of Cattle, Reflecting

Different Disease Phenotypes.

Front. Immunol. 12:729217. doi: 10.3389/fimmu.2021.729217

\section{Timing of Transcriptomic Peripheral Blood Mononuclear Cell Responses of Sheep to Fasciola hepatica Infection Differs From Those of Cattle, Reflecting Different Disease Phenotypes}

\begin{abstract}
Dagmara A. Niedziela ${ }^{1}$, Amalia Naranjo-Lucena ${ }^{1}$, Verónica Molina-Hernández ${ }^{2}$, John A. Browne ${ }^{3}$, Álvaro Martínez-Moreno ${ }^{4}$, José Pérez ${ }^{2}$, David E. MacHugh ${ }^{3,5}$ and Grace Mulcahy ${ }^{1,5 *}$
\end{abstract}

1 UCD School of Veterinary Medicine, University College Dublin, Dublin, Ireland, 2 Departamento de Anatomía y Anatomía Patológica Comparadas y Toxicología, Facultad de Veterinaria, Universidad de Córdoba, Córdoba, Spain, ${ }^{3}$ Animal Genomics Laboratory, UCD School of Agriculture and Food Science, University College Dublin, Dublin, Ireland, ${ }^{4}$ Departamento de Sanidad Animal (Parasitología), Facultad de Veterinaria, Universidad de Córdoba, Córdoba, Spain, ${ }^{5}$ UCD Conway Institute of Biomolecular and Biomedical Research, University College Dublin, Dublin, Ireland

Infection with the zoonotic trematode Fasciola hepatica, common in many regions with a temperate climate, leads to delayed growth and loss of productivity in cattle, while infection in sheep can have more severe effects, potentially leading to death. Previous transcriptomic analyses revealed upregulation of TGFB1, cell death and Toll-like receptor signalling, T-cell activation, and inhibition of nitric oxide production in macrophages in response to infection. However, the differences between ovine and bovine responses have not yet been explored. The objective of this study was to further investigate the transcriptomic response of ovine peripheral blood mononuclear cells (PBMC) to $F$. hepatica infection, and to elucidate the differences between ovine and bovine PBMC responses. Sixteen male Merino sheep were randomly assigned to infected or control groups ( $\mathrm{n}=8$ per group) and orally infected with $120 \mathrm{~F}$. hepatica metacercariae. Transcriptomic data was generated from PBMC at 0,2 and 16 weeks post-infection (wpi), and analysed for differentially expressed (DE) genes between infected and control animals at each time point (analysis 1), and for each group relative to time 0 (analysis 2). Analysis 2 was then compared to a similar study performed previously on bovine PBMC. A total of $453 \mathrm{DE}$ genes were found at $2 \mathrm{wpi}$, and $2 \mathrm{DE}$ genes at 16 wpi (FDR $<0.1$, analysis 1 ). Significantly overrepresented biological pathways at 2 wpi included role of PKR in interferon induction and anti-viral response, death receptor signalling and RIG-I-like receptor signalling, which suggested that an activation of innate response to intracellular nucleic acids and inhibition of cellular apoptosis were taking place. Comparison of analysis 2 with the previous bovine transcriptomic study revealed that anti-inflammatory response pathways which were significantly overrepresented in the 
acute phase in cattle, including $I L-10$ signalling, Th2 pathway, and Th1 and Th2 activation were upregulated only in the chronic phase in sheep. We propose that the earlier activation of anti-inflammatory responses in cattle, as compared with sheep, may be related to the general absence of acute clinical signs in cattle. These findings offer scope for "smart vaccination" strategies for this important livestock parasite.

Keywords: transcriptomics, sheep, PBMC, Fasciola, anti-inflammatory response

\section{INTRODUCTION}

Fasciola hepatica is a zoonotic trematode that affects livestock (sheep, cattle, goats) as well as humans. Human cases of fasciolosis occur mainly in Latin America, Africa and Asia, with approximately 2.5 million people affected worldwide (1, 2). Sporadic human cases also occur in Europe as a result of the consumption of raw vegetables such as parsley or watercress, grown on irrigated or flooded land (3).

F. hepatica infection is an important animal health issue in ruminants in endemic areas (4). In Ireland, F. hepatica seroprevalence is reported as $75.4 \%$ to $82 \%$ of dairy herds, and within-herd prevalence is estimated at $\leq 50 \%(5,6)$. In Northern Ireland, $63.15 \%$ cattle herds were positive for F. hepatica, with an overall animal level prevalence of $23.68 \%$ (7). Horses can also become infected, with a $9.5 \%$ prevalence of $F$. hepatica antibodies reported in a post-mortem survey of horses in Ireland (8). The cost of livestock infection includes productivity losses (e.g., meat, milk, wool) as well as reduced fertility and condemnation of livers as unfit for human consumption $(9,10)$. While F. hepatica infection in cattle leads to delayed growth and loss of productivity, acute infection in sheep can have more severe effects. The severe disease presentation in sheep is linked to a rapid acute response and can lead to sudden death (11). Anthelmintic resistance in F. hepatica populations is an increasingly important issue, with resistance to triclabendazole of particular concern $(12,13)$, as this is the only drug effective against early immature flukes. Hence, there has been significant attention given to developing a vaccine effective in protecting ruminants against fasciolosis. While promising vaccine trials have been conducted, repeatability and consistency has been an issue $(14,15)$.

The ruminant immune response to $F$. hepatica infection involves an increase in peripheral eosinophil numbers, alternative activation of macrophages, and suppression of Th1 responsiveness to the parasites themselves and to bystander antigens $(16,17)$. The transcriptomic response of peripheral blood mononuclear cells (PBMC) to F. hepatica in cattle has been characterised by apoptosis of antigen-presenting cells (APCs), liver fibrosis and a Th2 response, with TNF, IL1B, and DUSP1, APP, STAT3 and mir-155 as important upstream regulator genes leading to hepatic fibrosis and apoptosis of APCs or migration and chemotaxis of leukocytes (18). A balanced Treg-Th2 response was present in the acute phase with increased polarization towards a Th2 response during the chronic phase of infection.

The transcriptomic response of ovine PBMC has also been characterised previously. The response at 2 and 8 weeks post- infection (wpi) was associated with upregulation of the TGF- $\beta$ signalling pathway, the complement system, chemokine signalling and T-cell activation. Groups of genes were identified which were important for the immune response to the parasite and included those coding for lectins, proinflammatory molecules from the S100 family and transmembrane glycoproteins from the CD300 family. Early infection ( $2 \mathrm{wpi}$ ) was characterised by positive activation of $\mathrm{T}$ cell migration and leukocyte activation, while the in the later stage ( 8 wpi) lipoxin metabolic processes and Fc gamma Rmediated phagocytosis were among the upregulated pathways (19). Another study which looked at the ovine acute and chronic response at 1 and 14 wpi identified upregulation of TGF $\beta$ signalling, production of nitric oxide in macrophages, death receptor signalling and IL-17 signalling at 2 wpi, as well as Toll-like receptor (TLR) and p53 signalling in both the acute and chronic phase (20). The overall conclusions of these studies suggest a drive towards an anti-inflammatory response and tissue repair in $F$. hepatica infection in both sheep and cattle.

The objective of this study was to further investigate the pathways involved in the response of ovine PBMC to $F$. hepatica infection, and to compare those with bovine responses to aid in developing effective vaccine strategies.

\section{MATERIALS AND METHODS}

\subsection{Animal Trial Outline}

The animal trial has been described previously by NaranjoLucena et al. (21). Briefly, 19 male Merino sheep were obtained from a liver fluke-free farm at 9 months of age. The animals were all purchased into the research farm at the same time and were acclimatized for 3 months before the infections. All animals were tested monthly for parasite eggs by faecal sedimentation. Prior to challenge, all animals were tested for serum IgG specific for FhCL1 by an enzyme-linked immunosorbent assay (ELISA). Animals were housed indoors $\left(100 \mathrm{~m}^{2}\right.$ covered and $100 \mathrm{~m}^{2}$ uncovered facilities) and fed with hay and pellets and given water ad libitum. The infected and control animals were allocated in the same house (co-housed). Sheep were randomly allocated to the infected and control group, with 11 animals in the infected group, and 8 animals in the control group. However, 3 infected animals were later excluded from the study due to: 1) sample not being available at 16 wpi (not enough PBMC), 2) an animal identified with a potential pre-existing condition due to high eosinophils pre-infection (9.2\%), low haemoglobin (anaemic), and liver atrophy on pathology examination, and 3) a potentially 
mislabelled sample. This resulted in $n=8$ in both groups. At week 0 , eight sheep were orally infected with one dose of $120 \mathrm{~F}$. hepatica metacercariae of the Ridgeway (South Gloucester, UK) strain. Animals were monitored for 16 weeks, with faecal egg counts measured weekly starting from 7 wpi, and haematology measurements taken at $0,2,7$ and 16 wpi. PMBC were purified from blood samples taken at 0,2 and 16 wpi and used for the transcriptomic analysis (Figure 1A). Sheep were sacrificed at 16 wpi and liver fibrosis was evaluated on gross pictures of the visceral and diaphragmatic aspect of the livers after necropsy. The presence of tortuous whitish scars was scored as 1 (mild): 0$10 \%$ of the liver surface affected, 2 (moderate): $10-20 \%$ of the liver surface affected, 3 (severe): $20-30 \%$ of the liver surface affected and 4 (very severe): more than $30 \%$ of the liver surface affected. The experiment was approved by the Bioethics Committee of the University of Cordoba (code No. 1118) and conducted in accordance with European (2010/63/UE) and Spanish (RD 1201/2005) directives on animal experimentation. The number of experimental animals was set to 8 per group from a power calculation performed for a similar study conducted by our group in cattle by Garcia-Campos et al. (18).

\subsection{RNA Extraction From Peripheral Blood Mononuclear Cells (PBMC)}

Blood samples were collected in $10 \mathrm{ml}$ EDTA vacutainers (BD) from the jugular vein. Under a flow hood, $30 \mathrm{ml}$ of whole blood per animal was added into Falcon tubes and centrifuged at $1900 \times$ $g$ for $30 \mathrm{~min}$ without brake at $4^{\circ} \mathrm{C}$ to separate the buffy coat, red blood cells and plasma. The buffy coat was aspirated, added to a $15 \mathrm{ml}$ Falcon tube and diluted 1:1 with PBS. After resuspension, the diluted buffy coat was gently layered on the top of $4 \mathrm{ml}$ Ficoll Histopaque (Sigma-Aldrich) and centrifuged at $1900 \times g$ for $30 \mathrm{~min}$ without brake at $4^{\circ} \mathrm{C}$. Then, the buffy coat (PBMC) formed in the interphase between Histopaque and medium was aspirated. PBMC were washed once with $10 \mathrm{ml}$ sterile PBS-EDTA and centrifuged at $1100 \times g$ for $10 \mathrm{~min}$ at $4^{\circ} \mathrm{C}$. After discarding the supernatant, the cells were resuspended in $5 \mathrm{ml}$ of sterile ammonium chloride potassium, ACK buffer $\left(155 \mathrm{mM} \mathrm{NH}_{4} \mathrm{Cl}\right.$, $10 \mathrm{mM} \mathrm{KHCO}_{3}$ and $0.1 \mathrm{mM}$ ethylenediaminetetraacetic acid, EDTA) for $2 \mathrm{~min}$ at room temperature to lyse the erythrocytes. Then, sterile PBS-EDTA was added up to $14 \mathrm{ml}$ and centrifuged at $1100 \times g$ for $10 \mathrm{~min}$ at $4^{\circ} \mathrm{C}$. The supernatant was discarded, and $10 \mathrm{ml}$ of sterile PBS were added. At this step, PBMC were counted using Trypan blue for viability assessment. After counting, tubes were centrifuged at $1100 \times g$ for $10 \mathrm{~min}$ at $4^{\circ} \mathrm{C}$ and $\mathrm{PBMC}$ were resuspended in $1.5 \mathrm{ml}$ of sterile $\mathrm{PBS}$, divided into cryotubes and centrifuged at $900 \times g$ for $10 \mathrm{~min}$ at $4^{\circ} \mathrm{C}$. After the PBS was removed, $1 \mathrm{ml}$ of RNAlater (Sigma-Aldrich) was added per cryotube. The tubes were kept at $4^{\circ} \mathrm{C}$ overnight and stored at $-80^{\circ} \mathrm{C}$ until further assays.

Cell pellets were stored in RNAlater at $-80^{\circ} \mathrm{C}$ until RNA extraction. Prior to extracting RNA, $950 \mu \mathrm{l}$ of sample in RNAlater was mixed with $950 \mu \mathrm{PBS}$ and centrifuged at $3000 \times g$ for $5 \mathrm{~min}$. RLT buffer (Qiagen) was then added to the pellet and RNA was extracted using an RNeasy Plus Mini kit (Qiagen) as per the manufacturer's instructions. RNA was eluted in $35 \mu \mathrm{l}$ of RNAse-free water (Sigma).

RNA quality was assessed using 2100 Bioanalyzer RNA Nano chips (Agilent) as well as with a NanoDrop ${ }^{\text {TM }} 1000$ spectrophotometer (Thermo Fisher Scientific). Sample RIN values ranged between 7.3 and 9.9 (mean RIN value 9.1) and concentrations ranged from 56 - $450 \mathrm{ng} / \mu \mathrm{l}$ (mean RNA concentration $158 \mathrm{ng} / \mu \mathrm{l}$ ) (Supplementary Table S1).

\subsection{Library Preparation and Sequencing}

RNA was sent on dry ice to Novogene (Beijing, China) for library preparation and sequencing. RNA quality was re-checked on arrival using a 2100 Bioanalyzer (Agilent) and Nanodrop.
A

In vivo infection protocol
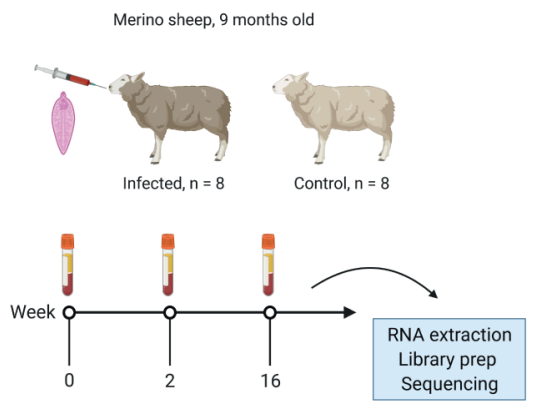

B

Bioinformatics analysis

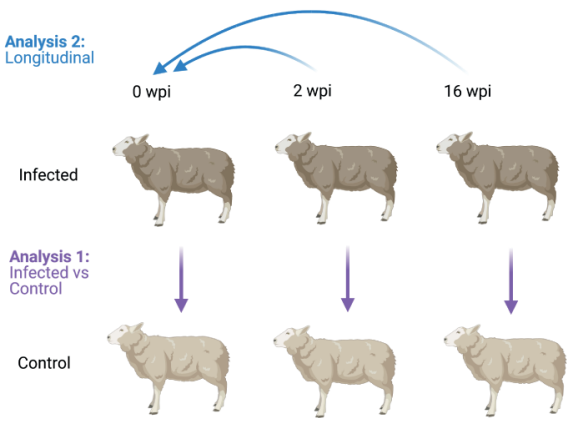

FIGURE 1 | Overview of the animal infection protocol (A) and bioinformatics analysis (B). Sixteen male Merino-breed sheep were randomly assigned to an infected or control group and orally infected with a single dose of liver fluke (Fasciola hepatica). Blood was then collected for isolation of peripheral blood mononuclear cells (PBMC). These cells were used to generate poly-A selected, stranded $2 \times 150$ bp RNA sequencing data on a NovaSeq 6000, at 0, 2 and 16 wpi. These data were then analysed for DE genes using two approaches: analysis 1 (infected vs control) - infected animals were compared to control animals at each time point; analysis 2 (longitudinal) - the infected and control animals were compared separately to their pre-infection time points. The DE gene lists of both groups were then compared, and the DE genes which occurred in the infected group but not in the control group at each time point were considered to be the potential DE genes in response to F. hepatica. wpi, weeks post-infection. Image created using (biorender.com). 
The RIN values as assessed by Novogene ranged from 7.7 to 10 (mean RIN value 9.45) (Supplementary Table S1). Stranded, poly-A selected mRNA library preparation was performed with approx. $800 \mathrm{ng}$ of RNA per sample, using a NEBNext ${ }^{\circledR}$ Ultra Directional RNA Library Prep Kit (New England BioLabs) according to the manufacturer's instructions. A 5400 Bioanalyzer (Agilent) was used to assess library fragment size distribution, and qPCR was used for library quantification. The average library fragment size was $230 \mathrm{bp}$ and ranged from $180 \mathrm{bp}$ to $280 \mathrm{bp}$. Pooled libraries were sequenced (150 bp, paired-end) on a NovaSeq 6000 (Illumina).

\subsection{Quality Control, Assembly and DE Gene Analysis}

All the bioinformatics pipeline bash and $\mathrm{R}$ scripts used for computational analyses were deposited in a GitHub repository at https://github.com/DagmaraNiedziela/RNAseq_Fhepatica_ ovine_PBMC. RStudio v1.3.1093 and R v4.0.3 were used for the analyses. Sequence quality was assessed using FastQC v0.11.8 (22). Trimming was performed using fastp v0.19.7 (23) with default settings as well as enabling base correction for paired end data, overrepresented sequence analysis, paired-end adapter removal, a minimum trimmed read length of 30 bases and a minimum phred score of 20 . On average, $1 \%$ of the reads were trimmed per sample. Trimmed fastq files were re-checked with FastQC to confirm sequence read quality. Trimmed sequences were mapped to the ovine genome (Rambouillet v1.0) using the STAR aligner v2.7.3a (24), with gene counts generated simultaneously with the STAR software using the Ensembl annotation v101 of the Ovis aries reference genome and annotation. Genes with read counts $<10$ across all samples as well as non-protein coding genes were removed in a filtering step (25). DeSeq2 v1.30.0 (26) was then used to visualise gene expression data and perform differentially expressed (DE) gene analysis. Data was normalised with a vst function and visualised with a principal component analysis (PCA) plot of the samples based on the 500 genes with the highest inter-sample variation. Two types of DE gene analyses were performed (Figure 1B). For Analysis 1 (Infected versus Control), DE gene analysis was performed using a negative binomial generalized linear model, with time and group as fixed effects, the individual animal included as a within-group effect, and a group by time interaction. Wald tests were performed to identify DE genes between infected and control animals at each time point, with $P$ values adjusted for multiple comparisons using the Benjamini and Hochberg (B-H) method (27). DE genes with an adjusted $P$ value $\left(P_{\text {adj. }}\right)<0.1$ were used for further data exploration and downstream analyses. In Analysis 2 (Longitudinal), raw counts of both the infected and control groups post-infection were compared to their day 0 values. Animal as batch effect was not included in this model, as animals from each group were considered as their own individual controls. A difference between the gene lists from infected and control group DE genes at each time point was examined as a potential response to F. hepatica. The longitudinal analysis was performed for comparison purposes, as several previous transcriptomic studies have used this approach.

\subsection{Pathway and Gene Correlation Analysis}

Significant $\left(P_{\text {adj. }}<0.1\right.$, primary analysis, $P_{\text {adj. }}<0.05$, longitudinal analysis) DE genes were converted to their one-to-one human orthologs using AnnotationDbi in R, ordered by adjusted $P$ value and analysed for enrichment of Gene Ontology (GO) terms, Kyoto Encyclopedia of Genes and Genomes (KEGG) and REACTOME pathways using gProfiler R package v0.7.0 (28). When a group of DE genes matched multiple pathways, the pathway that was represented by the highest number of DE genes was selected in a filtering step. Ingenuity Pathway Analysis (IPA; Qiagen) was also used to examine overrepresented pathways and predicted upstream and downstream transcriptional regulators.

\subsection{Cell Composition Analysis}

Transcripts per million (TPM) values were generated from raw gene counts using a custom R script. Ovine Gene IDs converted to one-to-one human orthologs were used for the analysis. To estimate the proportion of the different immune cell populations in each somatic cell sample, a quanTIseq algorithm which is based on expression of cell specific surface marker genes was used (29).

\section{RESULTS}

\subsection{Parasitological and Clinical Examinations}

Results of the parasitological examinations have previously been described by Naranjo-Lucena et al. (21). Briefly, no animals showed evidence of $F$. hepatica infection by faecal examination or specific antibody ELISA prior to experimental infection, and all remained clinically normal. After experimental infection, animals in the infected group showed the presence of $F$. hepatica eggs in their faeces by 9 wpi, and eggs were detected in all 8 infected animals by the end of the study. No eggs were detected in the control animals. Animals were euthanized at 16 wpi and at post-mortem examination no flukes were detected in control animals, while the mean fluke burden in infected animals was $48 \pm 11$ (SD) flukes per liver. At 2 wpi, infected animals showed an increased percentage of peripheral eosinophils compared to the control group. Haemoglobin concentrations were significantly lower in the infected group at 16 wpi and infected animals also exhibited a slower weight gain and enlarged hepatic lymph nodes (21). None of the uninfected sheep showed liver fibrosis. Five infected sheep showed mild hepatic fibrosis, two of them showed moderate hepatic fibrosis and one sheep showed severe hepatic fibrosis (Supplementary Table S1).

\subsection{Sequence and Mapping Quality}

PBMC collected pre-infection and at 2 and 16 wpi were used for 150 bp stranded paired-end sequencing. On average, $94 \%$ of the bases across all samples had a phred score of $\geq 30$ and $98 \%$ had a score of $\geq 20$. The mean number of reads per sample was $64.6 \mathrm{M}$. The distribution of read counts, phred scores and GC content per sample is available in Supplementary Table S1. Quality control, 
as assessed by FastQC, yielded satisfactory results for all samples. On average, $89.3 \%$ of reads mapped uniquely to the ovine genome Oar Rambouillet v1.0.

\subsection{Data Exploration}

Raw read counts were used for DE gene analysis using DESeq2. After filtering for low counts (sum of reads from all samples $<10$ ) and non-protein coding genes, 15,363 genes were retained for further analyses. The expression of the 500 most variable genes was used for generating PCA plots within the DESeq2 software package. Samples were observed to separate by time rather than by group, with 2 wpi and 16 wpi forming distinct clusters (Figure 2A). The largest sample variation was observed at time 0 , with samples dispersed across the PCA plot. This variation may reflect inter-animal differences being more pronounced at time 0 due to an acclimatisation period. For subsequent individual time points, the clearest separation between the infected and control groups was observed at 2wpi (Figure 2B).

\subsection{Cell Composition}

Normalised gene expression was used to elucidate proportions of different cell types in the PBMC at each time point. There were no major differences in cell populations at any time point (Supplementary Figure 1), which was in accordance with haematology parameters tested previously (21). Undefined cells did not differ between groups or time points and represented $55.3 \pm 0.8 \%$ in the control group and $54.8 \pm 3.1 \%$ in the infected group. These "other" undefined cells most likely represent CD4 and CD8 T lymphocytes. Both CD4 and CD8A were present in the data set and not differentially expressed, however the $C D 8 B$ gene was not found in the ovine genome annotation, which was

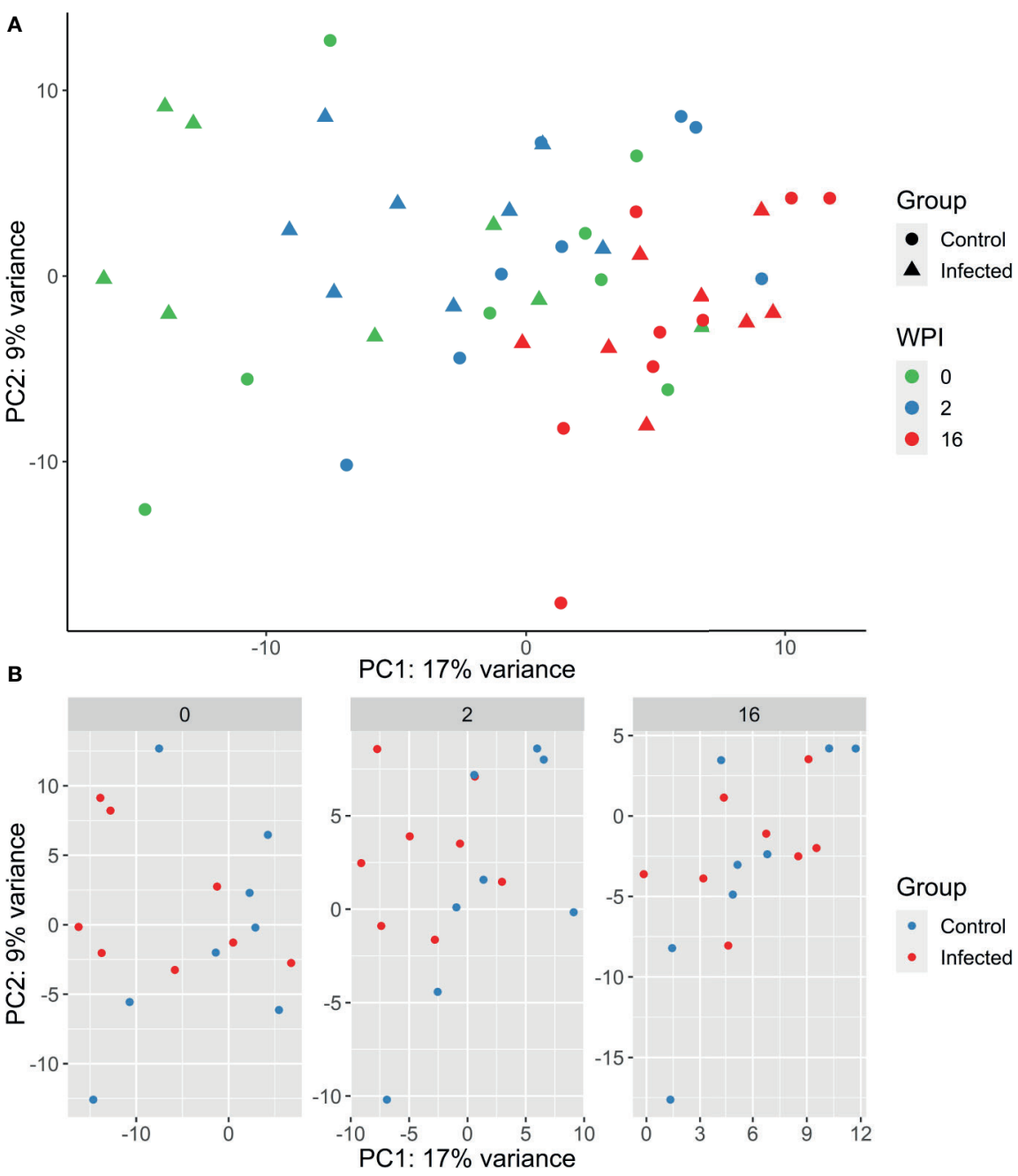

FIGURE 2 | (A) PCA plot generated from PBMC gene expression data of the 500 most variable genes following infection with $F$. hepatica in the infected (triangle) and control (circle) animals. Samples from each time point are represented by a different colour: 0 wpi - green, 2 wpi - blue, 16 wpi - red. WPI - weeks post infection. (B) PCA plots generated by time point, with infected animals in red and control animals in blue. 
likely the reason for CD8 T-cells not being identified by QuanTISeq. Numbers of T regulatory cells appeared higher in the infected group than in the control group at 2 wpi; however, the difference was not significant. Overall, lack of major differences in cell composition indicates that the response seen in this study is likely to be due to changes in gene expression by particular PBMC cell types, rather than by changes in predominant cell type.

\subsection{Differentially Expressed Genes}

Analysis of infected animals versus control animals (Analysis 1) yielded $59 \mathrm{DE}$ genes at $0 \mathrm{wpi}, 453 \mathrm{DE}$ genes at $2 \mathrm{wpi}$, and $2 \mathrm{DE}$ genes at 16 wpi $\left(P_{\text {adj. }}<0.1\right.$; Figure 3A). Only 7 of the 0 wpi DE genes overlapped with the 2 wpi DE genes, and these were removed from further pathway analysis (Figure 3B). The single DE gene which was common between 2 wpi and 16 wpi was HSPB8, which encodes the heat shock protein family B (small) member 8 , and was downregulated at both time points. A complete list of infected $v s$ control DE genes is included in Supplementary Table S2.

To assess differential expression in both groups over time, a longitudinal analysis (Analysis 2) was performed with infected or control group compared to time 0 at each post-infection time point ( 2 wpi vs 0 wpi and 16 wpi vs 0 wpi in the infected group, and the same in the control group). Due to a larger number of DE genes present, a $P_{\text {adj. }}$ value cut-off of 0.05 was used for this analysis. A total of $1522 \mathrm{DE}$ genes were detected in the infected group at 2 wpi, and $3949 \mathrm{DE}$ genes at $16 \mathrm{wpi}$. In the control group there were $769 \mathrm{DE}$ genes at 2 wpi and $2342 \mathrm{DE}$ genes at 16 wpi. Of the DE genes identified in the infected and control groups, 1148 were found only in the infected animals at 2 wpi, and 1927 at 16 wpi (Figure 3C). These 1148 and 1927 DE genes were used in further analyses. A comparison of the longitudinal Analysis 2 and the infected $v s$ control Analysis 1 revealed that only 33 genes were common between the two analyses at 2 wpi (Figure 3D).
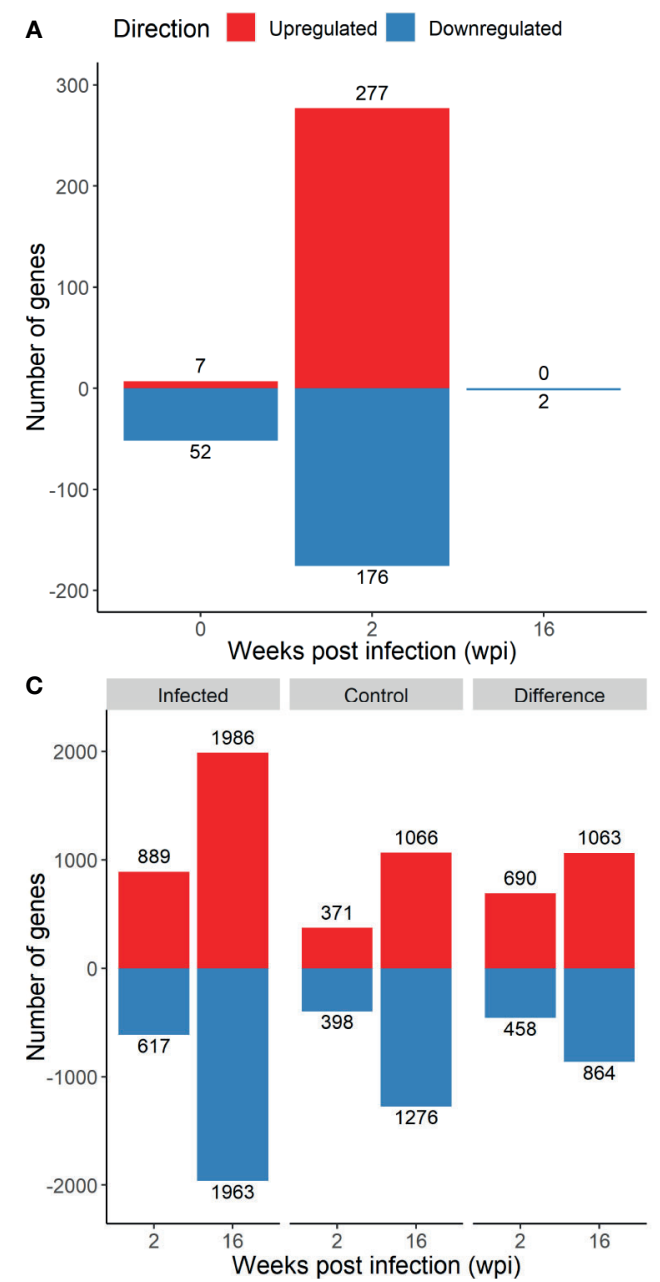

B

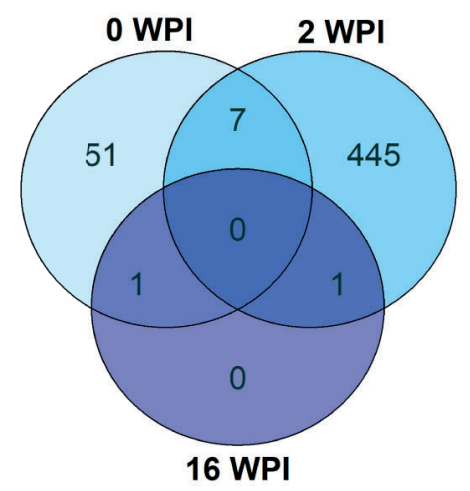

D

\section{Longitudinal Infected_vs_Control}

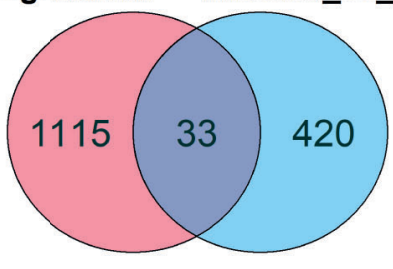

FIGURE 3 | Differentially expressed (DE) genes that were up- (red) and downregulated (blue) in PBMC in response to F. hepatica in the Infected vs Control analysis, with the Benjamini-Hochberg adjusted $P$ value $<0.1$ (A) and in the longitudinal analysis, adjusted P-value $<0.05$ (C). Venn diagrams of overlap of genes in the different time points of Infected vs Control analysis (B) and between the Longitudinal and Infected vs Control analysis at 2 wpi (D). 
In Analysis 1 (infected vs control), among the most significant upregulated DE genes in response to $F$. hepatica, the CD83 molecule gene (CD83) was a notable immune response gene in the top 5 at 2 wpi (Figure 4A). CD83 is a member of the immunoglobulin (Ig) superfamily and is expressed as membrane bound or soluble forms. Membrane bound CD83 is expressed by APC and is most highly and stably expressed by mature dendritic cells (DCs). Soluble CD83 has been reported to have an immune suppressive function, including a role in inhibition of DCs (30). Several uncharacterised genes were in the top 5 upand downregulated genes in the infected $v s$ control analysis, as well as the longitudinal analysis (Figure 4B), which may be reflective of the gaps in the functional annotation of the ovine genome.

Other notable infected $v s$ control analysis DE genes at 2 wpi involved in the immune response included: the complement $\mathrm{Clq}$ $\mathrm{A}$ and $\mathrm{B}$ chain genes $C 1 Q A$ and $C 1 Q B$; the $\mathrm{CD}$ molecule genes CD68, CD80 and CD151; heat shock protein genes HSPA13, HSPA5, HSPB1, HSPB8; mitogen-activated protein kinase (MAPK) genes MAP2K3, MAP3K1, MAPK11, MAPK1IP1L as well as genes associated with TNF, NF- $\kappa \mathrm{B}$ and interferon response (Table 1). Among these DE genes, TGIF1 was previously found to be downregulated in cattle at 1 wpi (18) in this study it was upregulated at 2 wpi.
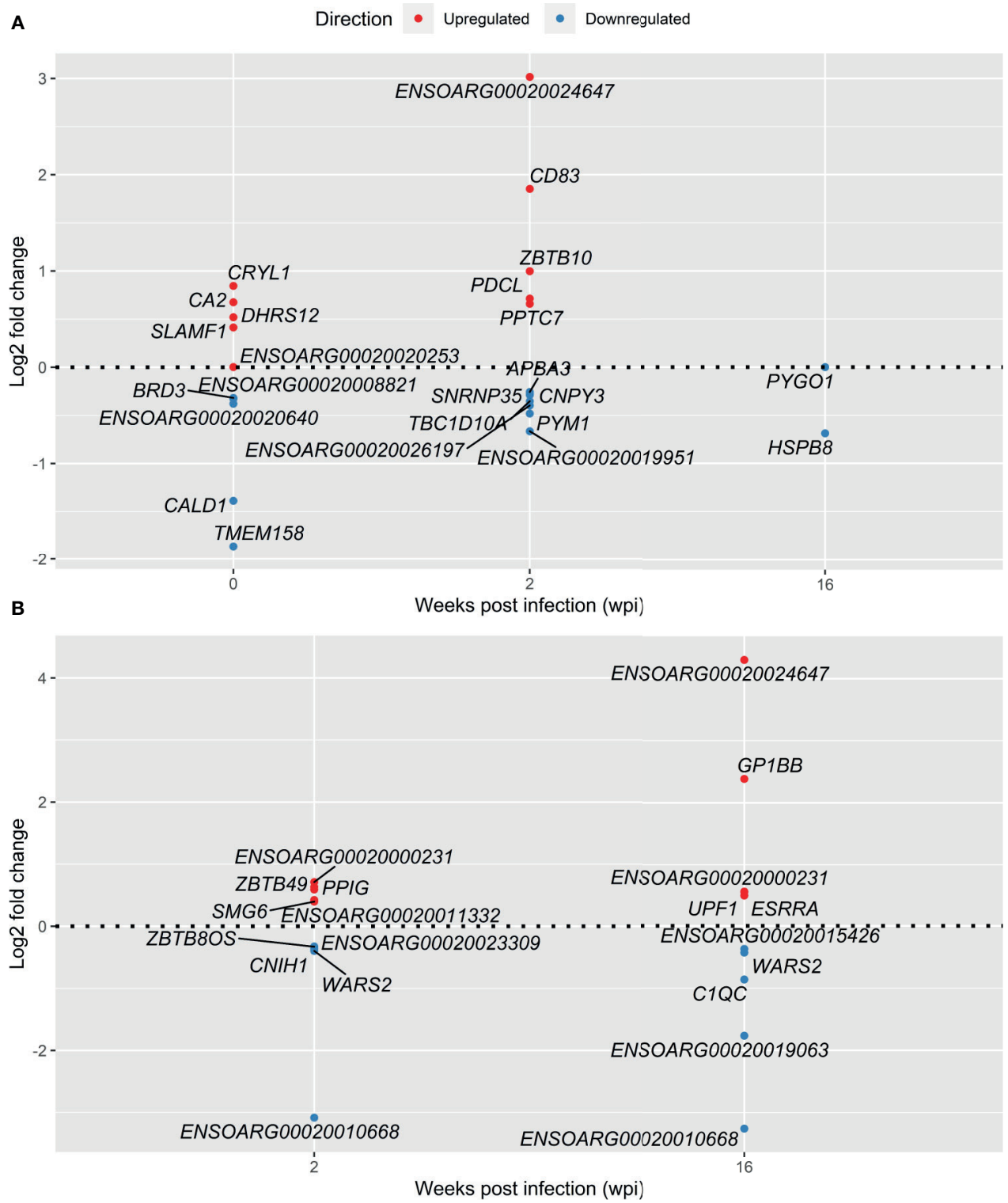

FIGURE 4 | Top 5 most significant up- and downregulated DE genes in response to infection with F. hepatica - Infected vs Control analysis (A) and Longitudinal analysis (B). Genes with lowest adjusted $P$ value were selected at each time point. In the case of genes with the same adjusted $P$ value both are included. 
TABLE 1 | Immune response-related DE genes at 2 wpi (infected vs control analysis).

\begin{tabular}{|c|c|c|c|}
\hline $\begin{array}{l}\text { Gene } \\
\text { Symbol }\end{array}$ & Gene Name & $\begin{array}{l}\log _{2} \text { Fold } \\
\text { change }\end{array}$ & $\begin{array}{l}\text { Adjusted } \\
P \text { value }\end{array}$ \\
\hline C1QA & complement C1q A chain & -0.60 & 0.04 \\
\hline$C 1 Q B$ & complement C1q B chain & -0.42 & 0.09 \\
\hline CASP8 & caspase 8 & -0.29 & 0.007 \\
\hline CASP9 & caspase 9 & -0.19 & 0.09 \\
\hline CD151 & CD151 molecule (Raph blood group) & -0.30 & 0.08 \\
\hline CD69 & CD69 molecule & 0.66 & 0.02 \\
\hline CD80 & CD80 molecule & 0.40 & 0.03 \\
\hline CD83 & CD83 molecule & 1.85 & 0.0005 \\
\hline CX3CR1 & C-X3-C motif chemokine receptor 1 & -0.86 & 0.01 \\
\hline DNAJA2 & $\begin{array}{l}\text { DnaJ heat shock protein family } \\
\text { (Hsp40) member A2 }\end{array}$ & 0.29 & 0.006 \\
\hline DNAJB4 & $\begin{array}{l}\text { DnaJ heat shock protein family } \\
\text { (Hsp40) member B4 }\end{array}$ & 0.80 & 0.03 \\
\hline HBEGF & heparin binding EGF like growth factor & 1.45 & 0.002 \\
\hline HSPA13 & $\begin{array}{l}\text { heat shock protein family } \mathrm{A}(\mathrm{Hsp} 70) \\
\text { member } 13\end{array}$ & 1.18 & 0.00009 \\
\hline HSPA5 & $\begin{array}{l}\text { heat shock protein family A (Hsp } 70) \\
\text { member } 5\end{array}$ & 0.35 & 0.01 \\
\hline HSPB1 & $\begin{array}{l}\text { heat shock protein family B (small) } \\
\text { member } 1\end{array}$ & -0.39 & 0.04 \\
\hline HSPB8 & $\begin{array}{l}\text { heat shock protein family B (small) } \\
\text { member } 8\end{array}$ & -0.45 & 0.03 \\
\hline IFNAR2 & $\begin{array}{l}\text { interferon alpha and beta receptor } \\
\text { subunit } 2\end{array}$ & -0.19 & 0.07 \\
\hline IL16 & interleukin 16 & -0.27 & 0.06 \\
\hline IRF9 & interferon regulatory factor 9 & -0.26 & 0.08 \\
\hline MAP2KЗ & $\begin{array}{l}\text { mitogen-activated protein kinase } \\
\text { kinase } 3\end{array}$ & -0.18 & 0.08 \\
\hline MAPЗK1 & $\begin{array}{l}\text { mitogen-activated protein kinase } \\
\text { kinase kinase } 1\end{array}$ & 0.46 & 0.02 \\
\hline MAPK11 & mitogen-activated protein kinase 11 & -0.59 & 0.05 \\
\hline MAPK1IP1L & $\begin{array}{l}\text { mitogen-activated protein kinase } 1 \\
\text { interacting protein } 1 \text { like }\end{array}$ & 0.23 & 0.09 \\
\hline NFKBID & NFKB inhibitor delta & 0.79 & 0.06 \\
\hline TGIF1 & TGFB Induced Factor Homeobox 1 & 0.25 & 0.09 \\
\hline TLR9 & toll like receptor 9 & 0.49 & 0.06 \\
\hline TNFAIP8L2 & TNF alpha induced protein 8 like 2 & -0.27 & 0.07 \\
\hline TNIP1 & TNFAIP3 interacting protein 1 & -0.14 & 0.06 \\
\hline TRAF3 & TNF receptor associated factor 3 & 0.30 & 0.08 \\
\hline TRAV26-2 & T cell receptor alpha variable $26-2$ & -0.84 & 0.01 \\
\hline
\end{tabular}

\subsection{Pathway Analysis of the Infected vs Control DE Genes}

Results of the infected $v s$ control DE analysis were used to investigate overrepresented gene pathways. HUGO Gene Nomenclature Committee (HGNC) gene symbols derived from the Ensembl annotation file for the Ovis aries Rambouillet v1.0 genome were used for pathway analysis using IPA and gProfileR, encompassing GO, KEGG, REACTOME and IPA databases. At 2 wpi, 60 out of 453 (13.24\%) DE genes had no HGNC assignment and were uncharacterised when queried using the Ensembl database. Key pathways that were overrepresented at 2 wpi included KEGG pathways such as RIG-I-like receptor (RLR) signalling, Toll-like receptor (TLR) signalling and protein processing in endoplasmic reticulum; and REACTOME pathways TLR Cascades and transcriptional regulation by TP53 (Table 2 and Supplementary Table S3). Significant IPA canonical pathways in the infected $v s$ control analysis at 2 wpi included role of $P K R$ in interferon induction and antiviral response, NRF2-mediated oxidative stress response, p38 MAPK signalling, B cell receptor signalling, death receptor signalling and protein kinase A signalling (Table 3 and Supplementary Table S4). There was a concordance between IPA and KEGG, since TLR signalling, role of RLRs in antiviral innate immunity, and protein ubiquitination were also among the significant IPA canonical pathways. Furthermore, examination of GO biological processes confirmed the findings of KEGG, REACTOME and IPA, notably since pathways related to stress, protein ubiquitination, apoptosis and cell death were overrepresented (Table 2).

Key groups of genes among the significant pathways were involved in response to $F$. hepatica at 2 wpi. For instance, in the transcriptional regulation by TP53 pathway, Polo-like kinase (PLK) genes PLK2 and PLK3 and BTG anti-proliferation factor 2 gene BTG2 were among the most upregulated genes (Figure 5A). In the TLR signalling pathway, the only upregulated TLR gene at 2 wpi was TLR9, and there was involvement of downregulated caspase gene CASP8, MAPK genes $M A P 2 K 3, M A P K 11$, and interferon alpha and beta receptor subunit 2 gene IFNAR2 (Figure 5B). At the same time, another MAPK gene (MAP3K1) was upregulated. In the RIG-I-like receptor signalling pathway the main antiviral receptor, retinoic acid inducible gene-I (RIG-I) encoded by $D D X 58$, as well as the main transcription factor, TNF receptor associated factor 3 gene TRAF3 were upregulated, possibly indicating pathway activation (Figure 5C). On the other hand,

TABLE 2 | Selected pathways overrepresented in response to F. hepatica at 2 wpi (infected vs control analysis), as identified by gProfileR.

\begin{tabular}{|c|c|c|c|}
\hline $\begin{array}{l}\text { Pathway } \\
\text { type }\end{array}$ & Pathway & $P$ value & $\begin{array}{l}\text { Number of } \\
\text { genes }\end{array}$ \\
\hline $\mathrm{BP}$ & response to organic substance & 0.0002 & 86 \\
\hline $\mathrm{BP}$ & cellular response to stress & 0.0003 & 46 \\
\hline $\mathrm{BP}$ & protein modification process & 0.0006 & 109 \\
\hline $\mathrm{BP}$ & response to abiotic stimulus & 0.001 & 28 \\
\hline $\mathrm{BP}$ & regulation of hemopoiesis & 0.005 & 20 \\
\hline $\mathrm{BP}$ & immune system development & 0.008 & 19 \\
\hline $\mathrm{BP}$ & apoptotic process & 0.01 & 24 \\
\hline $\mathrm{BP}$ & protein ubiquitination & 0.03 & 29 \\
\hline $\mathrm{BP}$ & adipose tissue development & 0.03 & 5 \\
\hline $\mathrm{BP}$ & negative regulation of cell death & 0.03 & 13 \\
\hline $\mathrm{BP}$ & hemopoiesis & 0.03 & 17 \\
\hline $\mathrm{BP}$ & cell death & 0.04 & 25 \\
\hline $\mathrm{BP}$ & negative regulation of cell communication & 0.04 & 34 \\
\hline KEGG & $\begin{array}{l}\text { Protein processing in endoplasmic } \\
\text { reticulum }\end{array}$ & 0.0001 & 13 \\
\hline KEGG & Herpes simplex infection & 0.02 & 12 \\
\hline KEGG & RIG-I-like receptor signalling pathway & 0.02 & 6 \\
\hline KEGG & Toll-like receptor signalling pathway & 0.05 & 8 \\
\hline REA & Circadian Clock & 0.00004 & 10 \\
\hline REA & Toll-Like Receptors Cascades & 0.003 & 13 \\
\hline REA & $\begin{array}{l}\text { Transcriptional regulation of white } \\
\text { adipocyte differentiation }\end{array}$ & 0.02 & 8 \\
\hline REA & MyD88-independent TLR4 cascade & 0.03 & 9 \\
\hline REA & TRIF(TICAM1)-mediated TLR4 signalling & 0.03 & 9 \\
\hline REA & Transcriptional Regulation by TP53 & 0.03 & 14 \\
\hline
\end{tabular}

$\overline{B P}$, biological process (gene ontology); KEGG, KEGG pathway; REA, REACTOME pathway. 
TABLE 3 | IPA canonical pathways in PBMC at the acute stage of $F$. hepatica infection in sheep as identified by the infected $v s$ control analysis, with a significance cut-off of $P=0.01$ (-log( $P$-value) of 2$)$.

\begin{tabular}{|c|c|c|c|}
\hline Ingenuity Canonical Pathways & $\begin{array}{l}-\log \\
(P \text { value })^{a}\end{array}$ & Ratiob $^{b}$ & z-score ${ }^{c}$ \\
\hline $\begin{array}{l}\text { Role of PKR in Interferon Induction and } \\
\text { Antiviral Response }\end{array}$ & 5.25 & 0.09 & -1.51 \\
\hline $\begin{array}{l}\text { D-myo-inositol }(1,4,5,6) \text {-Tetrakisphosphate } \\
\text { Biosynthesis }\end{array}$ & 4.49 & 0.08 & 1.27 \\
\hline NRF2-mediated Oxidative Stress Response & 4.00 & 0.06 & 0.71 \\
\hline p38 MAPK Signalling & 3.72 & 0.08 & 0.71 \\
\hline 3-phosphoinositide Degradation & 3.46 & 0.06 & 1.00 \\
\hline B Cell Receptor Signalling & 3.44 & 0.06 & 1.00 \\
\hline D-myo-inositol-5-phosphate Metabolism & 3.43 & 0.06 & 1.00 \\
\hline Unfolded protein response & 3.43 & 0.11 & 1.34 \\
\hline Endoplasmic Reticulum Stress Pathway & 3.41 & 0.19 & 1.00 \\
\hline Protein Kinase A Signalling & 3.26 & 0.04 & -1.07 \\
\hline 3-phosphoinositide Biosynthesis & 3.24 & 0.06 & 1.00 \\
\hline FGF Signalling & 3.24 & 0.08 & 0.82 \\
\hline $\begin{array}{l}\text { Superpathway of Inositol Phosphate } \\
\text { Compounds }\end{array}$ & 3.20 & 0.06 & 1.27 \\
\hline Endocannabinoid Cancer Inhibition Pathway & 3.11 & 0.06 & -1.67 \\
\hline Coronavirus Pathogenesis Pathway & 2.96 & 0.06 & -0.33 \\
\hline CD27 Signalling in Lymphocytes & 2.69 & 0.09 & 1.00 \\
\hline PEDF Signalling & 2.56 & 0.07 & 0.00 \\
\hline TWEAK Signalling & 2.55 & 0.11 & -1.00 \\
\hline April Mediated Signalling & 2.33 & 0.10 & NA \\
\hline Death Receptor Signalling & 2.31 & 0.07 & -0.82 \\
\hline B Cell Activating Factor Signalling & 2.29 & 0.10 & NA \\
\hline ATM Signalling & 2.20 & 0.06 & 0.45 \\
\hline $\begin{array}{l}\text { Role of RIG1-like Receptors in Antiviral Innate } \\
\text { Immunity }\end{array}$ & 2.18 & 0.09 & NA \\
\hline Protein Ubiquitination Pathway & 2.13 & 0.04 & NA \\
\hline ERK5 Signalling & 2.12 & 0.07 & 1.34 \\
\hline $\begin{array}{l}\text { Hypoxia Signalling in the Cardiovascular } \\
\text { System }\end{array}$ & 2.07 & 0.07 & NA \\
\hline Toll-like Receptor Signalling & 2.02 & 0.07 & NA \\
\hline GNRH Signalling & 2.01 & 0.05 & -0.378 \\
\hline
\end{tabular}

${ }^{a}-\log (P$ value $)$ - The probability of association of genes from our dataset with the canonical pathway by random chance alone using Fisher's exact test.

${ }^{b}$ ratio - The number of genes in our dataset that meets the cut-off criteria (FDR $<0.05$ and detected only in the infected group), divided by the total number of genes involved in that pathway.

${ }^{c} Z$-score - Overall activity status of the pathway. $Z<0$ indicates a prediction of an overall decrease in activity while $Z>0$ predicts an overall increase in the activity. NA indicates pathways that are currently ineligible for a prediction.

upregulation of the NFKB inhibitor delta gene NKFBID indicates possible downregulation of downstream cytokine production through the $\mathrm{NK}-\kappa \mathrm{B}$ pathway. The role of $P K R$ in interferon induction and antiviral response pathway involved DE genes present in the other pathways including CASP8, DDX58, IFNAR2, MAPK genes, NKFBID, TLR9 and TRAF3 (Figure 5D and Supplementary Figure 2). CASP9 and interferon regulatory factor 9 gene IRF9 were also downregulated in this pathway.

\subsection{Comparative Analysis of Longitudinal Bovine and Ovine PBMC Transcriptomic Response to F. hepatica}

A previous study by our group (18) described the bovine transcriptomic response to $F$. hepatica infection and found no robust evidence of DE genes when infected and control cattle were compared at acute and chronic timepoints post-infection. Therefore, a longitudinal analysis was performed in relation to time 0 , and the difference between infected and control DE gene lists were considered to be the DE genes in response to $F$. hepatica. A comparable longitudinal analysis was performed in this study, with the same number of infected and control animals in both studies $(n=8)$. To elucidate differences in responses of cattle and sheep, the longitudinal DE genes between this study and the study by Garcia-Campos et al. (18) were compared. In the bovine study, $21 \mathrm{DE}$ genes were found to differ between infected and control DE gene lists at $1 \mathrm{wpi}$, and $1624 \mathrm{DE}$ genes at 14 wpi (18), while $1148 \mathrm{DE}$ genes at 2 wpi, and 1927 at 16 wpi were detected in the current ovine study (Supplementary Table S5). The number of DE genes at the acute stage of infection in cattle was much lower than during chronic infection, while in sheep more than 1000 DE genes were detected at both the acute and chronic phases. This suggests that although in cattle penetration of immature flukes from the gut to the peritoneum elicits a relatively muted host response, the ovine response at the acute phase is more marked.

DE gene lists from the current ovine study and the bovine study were converted to human orthologs and gene lists at the acute and chronic stage were compared (Figure 6A). There were three common DE genes between cattle and sheep at the acute stage of infection: IL1RL1, CHD1 and RASSF1, and 232 common DE genes at the chronic stage of infection (Supplementary Table S6). When all time points in both species were compared, one DE gene was common to all time points in both sheep and cattle - the interleukin 1 receptor like 1 gene (IL1RL1). Common chronic DE genes included those involved in the action of TGF $\beta$, such as the TGFB induced factor homeobox 1 (TGIF1) and transforming growth factor beta receptor 3 (TGFBR3) genes. TGIF1 was downregulated in both species, while TGFBR3 was upregulated in cattle and downregulated in sheep. Proinflammatory cytokine and chemokine genes were also among the common chronic phase DE genes, including $C C L 18, C C L 2$, and $I L 1 B$, as well as the immune receptor genes CCRL2, IL10RA, IL2RG and TLR4. Several genes were common between the species, but exhibited opposing direction of expression (Figure 6B).

Pathway analysis of the longitudinal DE gene lists at acute and chronic phases was performed using IPA for both species. Three IPA canonical pathways were enriched in acute phases in both sheep and cattle, and eight canonical pathways were enriched in chronic phases for both species. The common acute pathways between sheep and cattle included Toll-like receptor signalling, PPAR activation, and RAR activation (Figure 7). In sheep two of these common pathways were overrepresented in both acute and chronic phases of infection. TREM1 signalling, STAT3 pathway and role of IL-17F in allergic inflammatory airway diseases were upregulated in both species at the chronic stage. CD28 signalling in T Helper cells, Phospholipase $C$ signalling and $N F-\kappa B$ signalling were upregulated in the chronic phase in sheep, but downregulated in cattle, while the hepatic fibrosis pathway was ineligible for prediction of activation/inhibition. A total of seven pathways were 
A

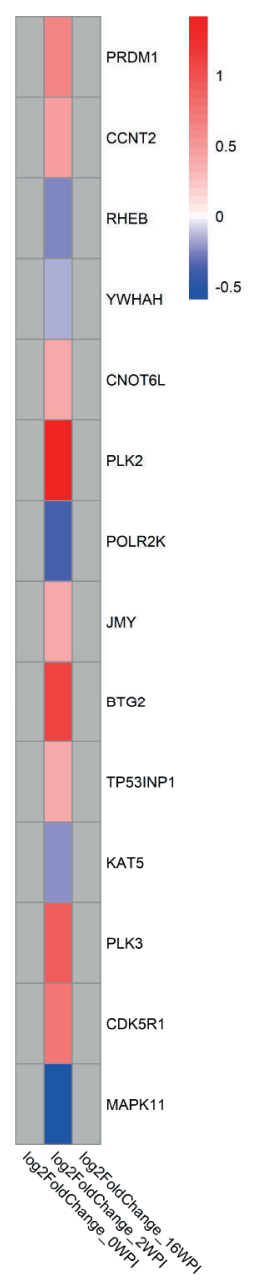

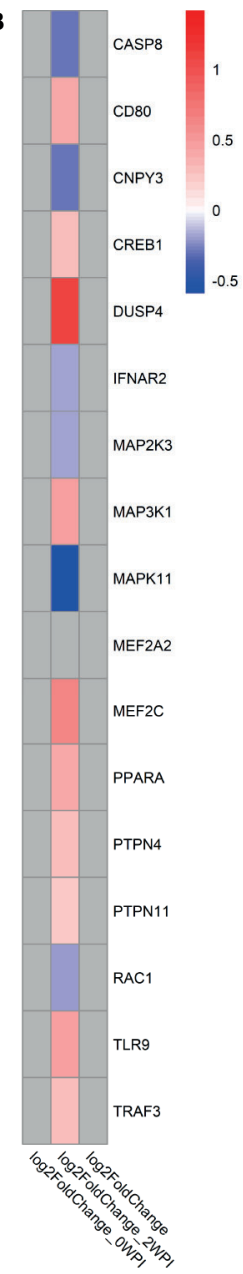

c

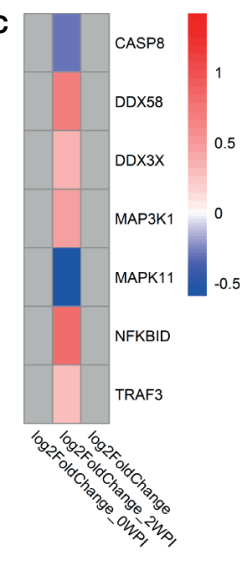

D

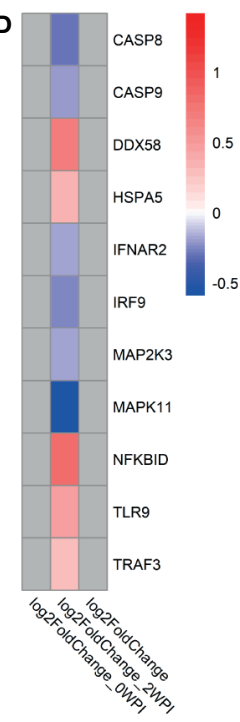

FIGURE 5 | Log $_{2}$ fold change of genes involved in selected KEGG, REACTOME and IPA pathways in response to F. hepatica (infected vs control analysis) at each time point. (A) Transcriptional Regulation by TP53 (REACTOME), (B) Toll-like receptor signalling (REACTOME, KEGG, IPA), (C) RIG-I-like receptor signalling pathway (KEGG,IPA), (D) The role of PKR in interferon induction and antiviral response (IPA). Grey tiles show genes that were not significantly DE. If pathways were found in multiple databases, gene lists were derived as a consensus of all genes in the pathway found in all databases.

significant in the chronic phase in sheep and in the acute phase in cattle, and included IL-10 signalling, IL-6 signalling, Th2 pathway and Th1 and Th2 activation pathway, which were all upregulated in sheep.

Many of the pathways specific to sheep were significant in both acute and chronic phases, and included EIF2 signalling, NGF signalling, NER Pathway, SAPK/JNK signalling and B Cell Receptor signalling (Supplementary Table S7).

\section{DISCUSSION}

F. hepatica is an important pathogen that causes zoonotic disease and economic losses in animal agriculture. Due to lack of acquired immunity following natural infection (31, 32) and anthelmintic resistance concerns, there is an urgent need for the development of novel control methods such as vaccination. Over the last few decades, progress has been made in the isolation, characterisation and testing of a number of native and recombinant molecules as vaccines against liver fluke disease in ruminant hosts (33). However, data from vaccine trials have been inconsistent (14), and some recent trials with recombinant antigens failed to induce protection against fluke infection (20) or achieved only a minor reduction of fluke burdens (34). In spite of evidence that effective vaccination may be possible, design of smart vaccines will require more knowledge about the immune response to $F$. hepatica, which can be provided by big-data approaches such as transcriptomics. This study provides an insight into the response of ovine PBMC to F. hepatica at both the acute and chronic stages of infection, and is the first long-term ovine PBMC transcriptomic study that includes uninfected control animals in the experimental design. 
A

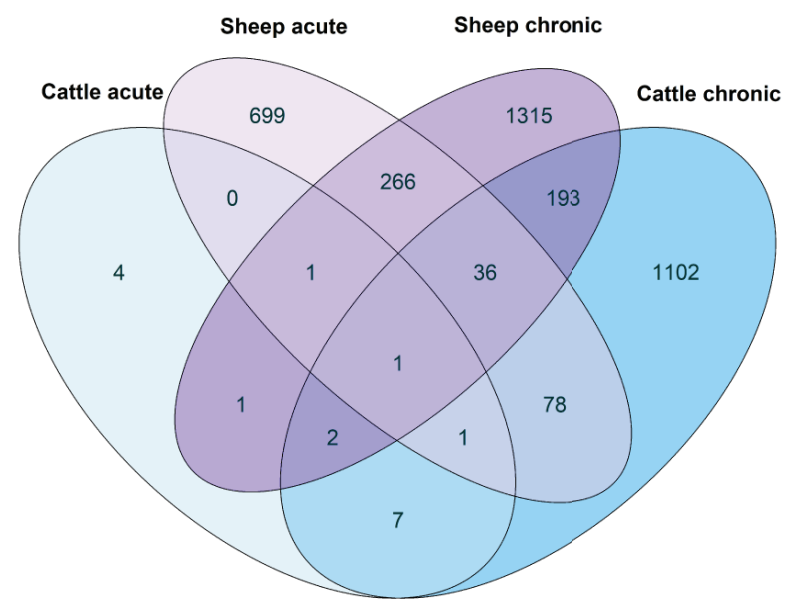

B
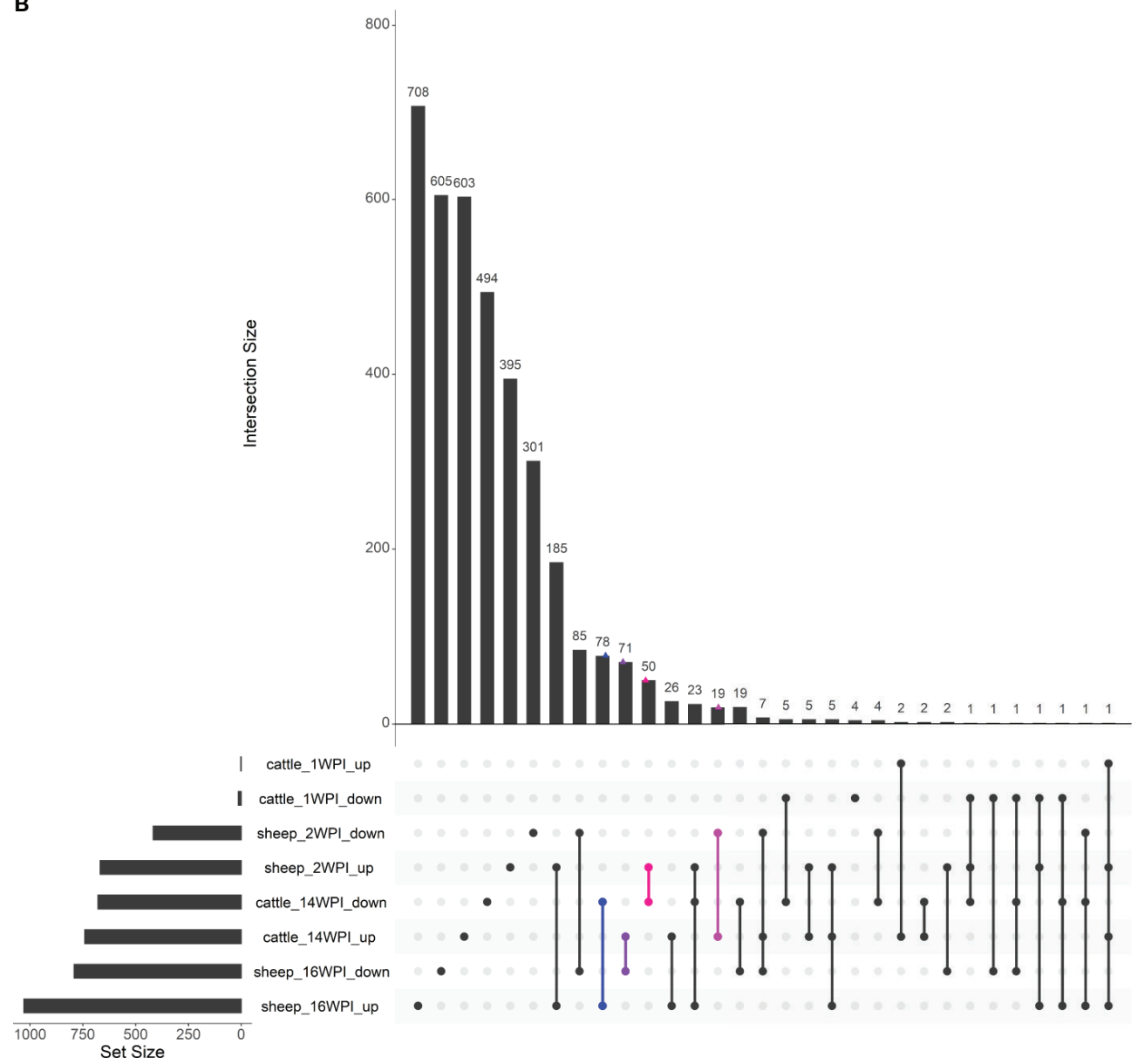

FIGURE 6 | Overlap of genes from the longitudinal analysis of cattle and sheep response to infection with $F$. hepatica between the acute and the chronic phase depicted by a Venn diagram (A) and an UpSet plot where the overlaps between up- and downregulated genes are shown in both sheep and cattle at all time points (B). On the UpSet plot, overlaps where up-regulated genes are the same as downregulated genes in another species are indicated in colour (blue, purple - DE genes with opposite direction of expression in sheep and cattle chronic phase; pink, magenta - DE genes with opposite direction of expression in acute phase in sheep and chronic phase in cattle). 


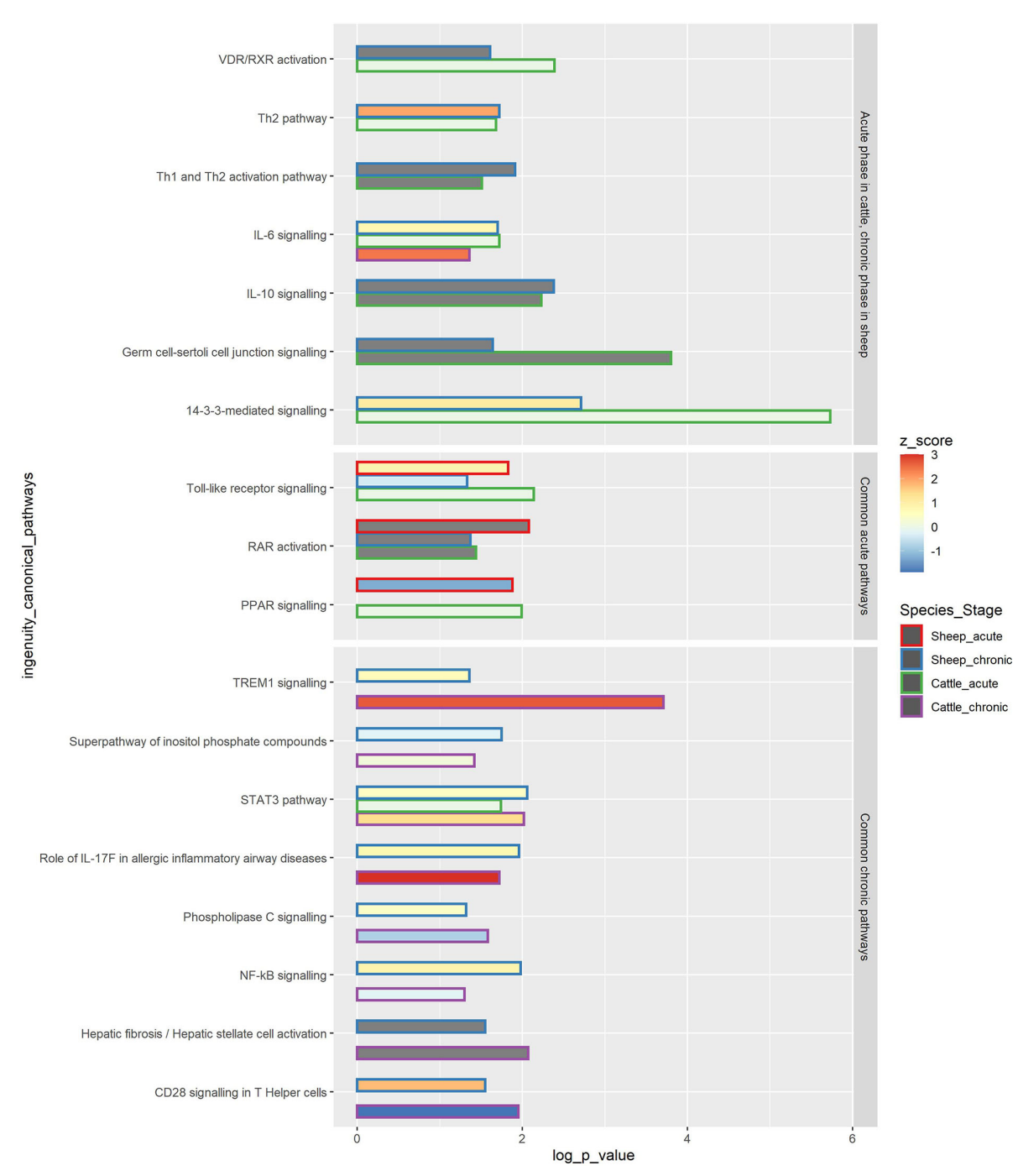

FIGURE 7 | Common IPA canonical pathways in PBMC at the acute and chronic stages of $F$. hepatica infection in sheep and cattle. The common pathways are subdivided into 3 squares: pathways occurring in the acute phase in cattle and in the chronic phase in sheep, common acute pathways and common chronic pathways. The length of the bar represents $-\log P$ value, colour within the bar represents the $z$ score, with the $z$ score of 0 being represented by a light green colour, and z score not determined represented by grey bar colour. The outline of the bar represents the species (sheep or cattle) and disease stage (acute or chronic).-log ( $P$ value) - The probability of association of genes from our data set with the canonical pathway by random chance alone using Fisher's exact test. Z-score - Overall activity status of the pathway. $Z<0$ indicates a prediction of an overall decrease in activity while $Z>0$ predicts an overall increase in the activity. Grey colour of the bar indicates pathways that are currently ineligible for a prediction.

\subsection{Infected vs Control Analysis}

\subsubsection{RIG-I Like and Toll-Like Receptor Signalling Pathways Are Identified by Multiple Databases and Indicate Potential Production of Interferons and Downregulation of Apoptosis}

Two databases (KEGG and IPA) identified the RLR signalling pathway as significant, and TLR signalling was significantly overrepresented in 3 databases (KEGG, REACTOME and IPA).

\subsubsection{RIG-I Like Receptor Signalling}

RLRs are cytosolic RNA helicases that recognise viral RNA inside immune and non-immune cells, leading to recruitment of intracellular adaptor proteins and ultimately to production of type I interferon and inflammatory cytokines. RLRs include RIGI, melanoma differentiation-associated gene 5 (MDA5), and DExH-box helicase 58 (DHX58 or LGP2) (35). The key receptor in this pathway, RIG-I, is encoded by the DDX58 gene, which was upregulated in this study at 2 wpi (Figure 5C). RIG-I is responsible for the innate recognition of uncapped 5'-phosphate double-stranded or single-stranded RNA found in numerous viruses including influenza and Ebola (35). Another key gene that was upregulated in our study and encodes an important transcription factor is the TRAF3 gene. Since TRAF3 is key to intracellular responses, molecules secreted by 
the parasite may undergo endocytosis and be detected while inside the cell. Therefore, the RLR signalling pathway could be involved in recognition of and response to extracellular vesicles of F. hepatica, which can contain RNA and miRNA and are secreted throughout the life cycle of the parasite, including newly excysted juveniles and adults $(36,37)$. TRAF3 activity in RLR signalling is further regulated by the action of Polo-like kinases (38), which were also upregulated at 2 wpi (Figure 5A). Overall, the upregulation of these key genes in the RLR signalling pathway would ultimately lead to an increase in interferon production. While no interferon genes were detected as upregulated in this study, the activation may occur at a later stage in the infection.

\subsubsection{Toll-Like Receptor Signalling}

TLR signalling was identified using both the KEGG and REACTOME pathway databases. The only TLR gene that was upregulated in this pathway was TLR9 (Figure 5B), which is activated by unmethylated CpG sequences in DNA molecules due to cancer, infection, or tissue damage. TLR9 is the receptor involved in the response to oncogenic viruses, and in autoimmune disorders. The overexpression of TLR9 coinciding with overexpression of DDX58 (RIG-I) further suggests that a response to nucleic acids secreted by $F$. hepatica is taking place. TLR7 and TLR9 are selectively expressed by plasmacytoid dendritic cells (pDCs; also known as interferon (IFN)producing cells), which are a subset of DCs with a plasmacytoid morphology and unique in their capacity to rapidly secrete large quantities of type I IFN in response to viral infection, including response to influenza (39). Glycans produced by $F$. hepatica were previously reported to induce a semi-mature state of DCs in mice, with reduced IFN- $\gamma$ production (40). Excretory-secretory products (ESP) from $F$. hepatica have also been found to inhibit the production of effector molecules such as IL-6 and IL-12p70 in DCs following stimulation of TLR9 with CpG (41). The DC receptor genes CD80 and CD83 were among the most upregulated DE genes in this study at 2 wpi (Table 1 and Figure 4A) - which may further suggest initial activation of DCs in response to F. hepatica, with further downstream response being inhibited by the parasite.

The lack of differential expression of other TLR genes in this study is in contrast to a previous study of transcriptomic response of ovine PBMC conducted by our group, where TLR1, TLR5, TLR6, TLR7 and TLR10 were downregulated in PBMC at the acute stage of infection (20). However, in a separate study of ovine PBMC, no TLR genes were DE at 2 wpi (19). TLR signalling also involves upregulation of TRAF3, which undergoes endocytosis together with TLR4 in response to lipopolysaccharide (LPS). LPS has been identified as a predicted upstream regulator by IPA at 2 wpi (results not shown), which may indicate a potential influence of helminth-derived LPS (perhaps from endosymbiotic bacteria) on the ovine response. Various other helminths have been demonstrated to possess gut endosymbionts that are transferred across generations and not present in the host's gut, including Neorickettsia in Plagiorchis elegans and Weissella and Leuconostoc in Haemonchus contortus (42). Neorickettsia has also been found in F. hepatica obtained from ovine liver (43). Helminth microbiomes could play a role in developmental variation and evolutionary fitness of the parasites and may influence the host immune response.

Ultimately, the TLR signalling pathway leads to upregulation of NF- $\kappa B$ and MAPK related genes. In this study, the NFKB inhibitor delta gene NFKBID was upregulated, and MAP kinases downregulated (Figure 5B), both of which would lead to a decreased cytokine response. In fact, we did not detect any interleukin genes to be DE apart from IL16, which points to an inhibition of cytokine response taking place.

Caspase 8 is a common protein in both RIG-I and TLR signalling pathways and is known to be a key driver of cellular apoptosis (44). This caspase was among the most downregulated DE genes at 2 wpi and suggests a potential inhibition of cellular apoptosis taking place at the acute stage of infection. Caspase 8 also may be key to the inflammatory response as $\operatorname{cas} p 8^{-/-}$mice exhibited a reduction in $I L 1 B$ and $I L 12 B$ expression, reduced recruitment of monocytes and an increased susceptibility to Toxoplasma gondii (45).

\subsubsection{Several Pathways Related to Apoptosis and Cell Death Were Identified}

\subsubsection{Transcriptional Regulation by TP53 Was} Overrepresented

Transcriptional regulation by TP53 is a REACTOME pathway that groups the upstream and downstream effects of the transcriptional regulator p53, most known for being involved in cancer regulation. The transcription factor recognizes specific responsive DNA elements and regulates the transcription of genes involved in cellular metabolism, survival, senescence, apoptosis and the DNA damage response $(46,47)$. PLK2, PLK3 and BTG2 were the most upregulated genes in the pathway (Figure 5A), encoding the PLK2 and 3 and BTG antiproliferation factor 2 proteins, respectively. PLKs play pivotal roles in cell cycle progression. PLK2 and 3 are activated by spindle checkpoints and DNA damage, and are required for entry into S phase (48). The fact that the PLK2 and PLK3 genes are highly upregulated here would indicate a progression of the cell cycle and activation of cell proliferation. BTG2 belongs to the anti-proliferative (APRO) family of genes that regulate cell cycle progression by enhancing or inhibiting the activity of transcription factors. The BTG2 gene has a potential role in muscle fibre size, intramuscular fat deposition and weight loss in sheep and can lead to a decrease in cell proliferation or an increase in energy expenditure (49). Therefore, upregulation of this gene may be related to increased cellular metabolism.

Other DE genes at 2 wpi that may indicate perturbation of the cell cycle include the cyclin D3, L1, Q and T2 genes (CCND3, CCNL1, CCNQ, CCNT2) and cyclin-dependent kinase genes CDK17 and CDK5R1, with the majority of these being upregulated (Supplementary Table S2). The product of the CCND3 gene, downregulated in this study, is responsible for the progression through the G1 phase (50). Cyclin types L and T, which were upregulated in this study, are involved in transcription $(51,52)$. It is therefore likely that transcription is activated at 2 wpi rather than PBMC proliferation. 


\subsubsection{The Role of $P K R$ in Interferon Induction and Antiviral Response Pathway Was Inhibited at 2 wpi} Protein kinase receptor (PKR) belongs to a family of patternrecognition receptors (PRRs). It can be induced by interferons (IFNs) as well as dsRNA from viral sources and responds by stimulating apoptosis of the host cell or by inhibition of translation, affecting both viral and cellular mRNA. Apoptosis can be activated via death receptor pathways such as caspases, as well as by direct phosphorylation of p53. Much of PKR signal transduction, however, occurs via NF- $\mathrm{KB}$ and MAPK pathways, which trigger various antiviral effects especially the production of type I IFNs and increased expression of pro-apoptotic factors (53). The interferon alpha and beta receptor subunit 2 (IFNAR2) and interferon regulatory factor 9 (IRF9) genes were downregulated in this pathway (Supplementary Figure 2). IRF9 is a transcription factor which mediates signalling by type I IFNs (IFN- $\alpha$ and IFN- $\beta$ ). Following type I IFN binding to cell surface receptors such as IFNAR, STAT1 and STAT2 are phosphorylated and IRF9 associates with the phosphorylated STAT1:STAT2 dimer to form a complex termed ISGF3 transcription factor, which then enters the nucleus and activates the transcription of interferon stimulated genes thereby driving the antiviral response of the cell (54). Downregulation of these genes at $2 \mathrm{wpi}$, indicates that the interferon response is inhibited. The NFKB inhibitor delta gene (NFKBID) is upregulated, which suggests inhibition of the PKR signal transduction that occurs through the NF- $\mathrm{KB}$ pathway. MAPK genes are downregulated, which also suggests inhibition of the PKR signal transduction which occurs through the MAPK pathway. Overall, the downregulation of this pathway suggests that an inhibition of innate immune response and cellular apoptosis are taking place. These mechanisms may facilitate host defence against $F$. hepatica, but be inhibited due to the parasite's immunomodulatory properties (55). Notably, anti-viral/small RNA response activation is suggested by upregulated genes in the TLR and RIG-I signalling pathways. This leads to the hypothesis that downstream interferon response is actively inhibited, even though foreign nucleic acids are being detected inside the cell.

\subsubsection{Death Receptor Signalling Was Inhibited at 2 wpi}

Death receptor signalling and apoptosis signalling were observed to be upregulated in the acute stage of $F$. hepatica infection in a study we conducted in sheep previously (20). In this study, the IPA death receptor signalling pathway was inhibited. Caspase-8 is a major initiator in the death signalling pathway (44). Previously, it has been shown that $F$. hepatica ESPs induced apoptosis of eosinophils, and caspase 3, 8 and 9 were activated in the process (16). CASP8 and CASP9 were both downregulated in this study, and CASP8 downregulation has also been described previously in sheep (20). Overall, the death receptor signalling pathway was inhibited; however, the key genes involved in the pathway such as TNF and CASP3 were not DE. This pathway should therefore be studied in more detail in order to elucidate the mechanisms of cell survival or apoptosis during infection with $F$. hepatica.

Overall, the pathways observed to be perturbed at 2 wpi in the infected $v s$ control analysis suggest that immune response to exogenous nucleic acids as well as changes in energy metabolism and cell viability occurred in the acute phase of ovine $F$. hepatica infection. Involvement of p53 related proteins, and a RIG-I like and TLR9 mediated anti-viral response are among the new response characteristics identified in this study.

\subsection{Longitudinal Analysis}

\subsubsection{Th2 Cell Activation}

In cattle, a shift towards Th2 responses highlighted by involvement of Th2 response and IL-4 and IL- 6 signalling pathways occurred in the acute phase of infection (18). It seems that the involvement of a Th2 response and antiinflammatory pathways involving IL-10 occurs only in the chronic phase in sheep, while in cattle these responses are activated much earlier. Activation of Th2 cells and inhibition of Th1 cells and a corresponding shift towards antiinflammatory pathways was also observed in a study we conducted on ovine hepatic lymph node (HLN) at $16 \mathrm{wpi}$, confirming that the anti-inflammatory responses are activated in sheep in the chronic phase of F. hepatica infection (21). This may suggest that it is the early activation of an anti-inflammatory response that causes lack of clinical signs in the acute infection in cattle, and that severe acute clinical signs in sheep are mainly caused by an acute inflammatory reaction in the attempt to remove the pathogen, with a corresponding lack of antiinflammatory response to mediate tissue damage. A tissue repair type of response seems effective in preventing severe disease in cattle, but not in providing protection against reinfection $(56,57)$.

\subsubsection{TLR Signalling}

The TLR pathway was upregulated in sheep in the acute phase and downregulated in the chronic phase of infection, as indicated by the IPA $z$ score (Figure 7 and Supplementary Table S7). On the other hand, TLR signalling was overrepresented (with no consensus on activation or inhibition, $z$ score $=0$ ) only at the acute stage in cattle (Figure 7). In cattle, the TLR2 and TLR4 genes were upregulated in the chronic phase, and TLR10 was downregulated (even though the pathway was not significant at the time), and no TLR genes were DE in the acute phase (18). On the other hand, in sheep, TLR4 was downregulated in the chronic phase, and no TLR genes were DE in the acute phase, with IL1RL1, LY96, and MAP4K4 being the main genes contributing to the activation of this pathway in both stages. TLR2 recognizes a wide variety of PAMPs including lipoproteins, peptidoglycans, lipoteichoic acids, zymosan, mannan, and tGPI-mucin, while TLR4 recognizes LPS, and TLR10 has been found to recognise ligands from Listeria and influenza A infections (58).

\subsubsection{Hepatic Fibrosis}

The IPA hepatic fibrosis pathway was overrepresented at the chronic stages in both sheep and cattle (Figure 7). TGF- $\beta 1$ is an essential cytokine promoting collagen production and fibrosis, which then leads to encapsulation of flukes and limiting migration of the parasites through the liver parenchyma (11, 59). Liver fibrosis in ruminants has previously been associated with expression of $I L 10$ and TGFB, with an increased expression 
of these genes potentially leading to increased fibrosis and control of fluke burdens (60). In cattle, liver fibrosis has been proposed as a downstream effect of upregulated TNF and $I L 1 B$, which would activate the genes that were also upregulated in the bovine study, i.e., IL6, PLAU, SERPINE1, TNFRSF1A, SOCS1, and CTSB (18). In sheep, however, the pathway was observed to exhibit a more recognisable outcome, with the involvement of IFNG, the collagen genes COL11A1 and COL11A2, and matrix metalloprotease MMP9 and SMAD2 genes (Supplementary Table S7). Even though typically collagen type I and III are involved in chronic liver fibrosis, the upregulation of genes encoding alpha and beta subunits of collagen type XI as well as a matrix metalloprotease gene, which is involved in reorganisation of the extracellular matrix, supports a potential role of these proteins in fibrosis in chronic liver fluke infection in sheep. Interferons alpha and gamma, typically secreted by NK cells, are also thought to inhibit liver fibrosis $(61,62)$. IFNG was downregulated in the chronic phase in sheep, which further supports activation of fibrosis. Surprisingly, SMAD2 was downregulated (Supplementary Table S6), which could suggest inhibition of fibrosis, even though other genes in the pathway were activated. IL1B and TLR 4 were involved in the pathway in both sheep and cattle, so there could also be a role in liver fibrosis activation for IL1B. TNF was not DE in sheep. The SERPINE1 gene, which was detected at chronic stages in the bovine study (18) and in a previous study of ovine PBMC response following infection with $F$. hepatica (20), was also not DE in this study.

The low number of DE genes in the acute response of cattle, coupled with specific anti-inflammatory pathways such as Th2 activation or IL-10 signalling, which were upregulated during the chronic phase in sheep and that were upregulated in the acute phase in cattle, underscore the significance of the acute response in sheep and its potential detrimental effect on disease phenotype. It is important to note that in sheep, following experimental infection, flukes mature simultaneously. Therefore, sheep typically yield adult flukes of approximately the same age and size in the liver during pathological examination at the chronic stage, while in cattle the chronic infection presents with a mix of juvenile and adult flukes. The more gradual progression of flukes to maturity in cattle suggests that the interaction between the parasite and the host may cause a delay in fluke maturation, potentially by inhibiting the growth of neoblasts (63). On the other hand, sheep do not seem to be able to slow the life cycle of the fluke, which then causes a rapid migration of the flukes through the gut and maturation in the liver causing a large inflammatory response, coupled with an attempt to repair tissue damage and slow inflammation through Th2 responses. The conclusion, therefore, would be that an early inflammatory response to $F$. hepatica, as seen in sheep $(11,64$, 65), has major pathological consequences.

\subsubsection{Comparison With a Previous Ovine PBMC Study by our Group}

Garcia-Campos et al. (18) discussed their findings in comparison with a previous transcriptomic study of ovine PBMC by Fu et al. (20) and concluded that in sheep, the majority of DE genes were present in the acute phase when compared to the chronic phase, while in cattle only $5 \%$ of the total DE genes were expressed in the acute phase of infection. While the proportion of acute DE genes in the bovine response is indeed very low, our study shows that using the same longitudinal analysis as in the cattle transcriptomic study, with the inclusion of control animals in the analysis, leads to a result where $63 \%$ of all DE genes were allocated to the chronic phase. Even when examining the infected animal group only, as in the study by Fu et al. (20), the majority of DE genes in our study were expressed during the chronic phase of infection, with $72 \%$ of the DE genes detected at this stage. It is important to note, however, that in the previous ovine transcriptomic study by Fu et al. (20) the DE genes at the chronic phase were compared to the acute phase (i.e., T3 vs T2), not to the baseline of week 0 . In the bovine DE gene analysis, the number of DE genes from the chronic phase compared to acute phase (WK14 vs WK1) was three-fold lower than when the chronic phase was compared to baseline (18). Therefore, the choice of time point comparison in the DE gene analysis could explain the skew towards the acute phase in the previous study of the transcriptomic response in sheep. Breed differences, as well as a differences in the F. hepatica strain used could also partially account for the different results when comparing our study to $\mathrm{Fu}$ et al. (20).

In both our study and the bovine study by Garcia-Campos et al. (18) the $\log _{2}$ fold changes of DE genes were relatively low in comparison to the previous ovine transcriptomic study (20): in our study the longitudinal DE gene $\log _{2}$ fold changes ranged from -3.1 to 3.3 in the acute phase, and from -3.2 to 4.3 in the chronic phase. While these $\log _{2}$ fold changes are still higher than in the bovine study, the differences are relatively minor, especially given the different DE gene analysis packages used (edgeR in the bovine study vs DEseq2 in the current ovine study), which could lead to slightly different results (26). The previous ovine study (20) observed much larger $\log _{2}$ fold changes, ranging from -27 to 12 . The study used a Limma-Voom package for DE gene analysis, which could at least partially account for the differences in $\log _{2}$ fold changes. The DE gene analysis in Limma is based on a linear model, with gene counts typically log-transformed, while DESeq2 and edgeR use a negative binomial generalised linear model, with raw counts used for the analysis. A difference in normalisation of raw counts prior to $\mathrm{DE}$ gene analysis could also cause differences in $\log _{2}$ fold changes.

\subsection{Comparison With Previous Studies}

Our study confirmed the need to use control animals in the infection. A greater number of DE genes were identified in sheep in response to F. hepatica by $\mathrm{Fu}$ et al. (20). However, this previous study did not use uninfected control animals in its design, and DE genes were compared to pre-infection. In fact, when the DE gene analysis was performed in this study in comparison to time 0 (longitudinal analysis), a large number of $\mathrm{DE}$ genes was identified in both infected and control animals, a phenomenon also found in a previous bovine study (18). This suggests that presence of $\mathrm{DE}$ genes related to developmental changes that occur in 9-month-old sheep could obscure the response to infection. Therefore, a study on older animals may be 
warranted, where growth effects may not be so dominant. Another possible explanation may be the seasonality of the infection and the onset of low temperatures. A previous study by Liu et al. (66) identified DE genes in sheep due to wool production between the months of August and December. The resulting DE genes included IL1B, IL5, IL8 and CXCL1, which suggests that immune proteins may be produced or inhibited due to seasonal changes. Our study was conducted between September and January, which could suggest a similar seasonal effect. In addition, immune response in sheep could be breeddependent, as demonstrated by relative resistance of the Indonesian thin tail sheep breed against $F$. gigantica when compared to Merino sheep $(67,68)$. Our study was conducted on Merino sheep, while the previous ovine study (20) used the Suffolk breed. Longitudinal studies of gene expression in sheep with seasonal changes, potentially in conjunction with measuring hormones and other physiological variables could assist future transcriptomic studies of host response.

In a previous ovine PBMC study by Alvarez Rojas et al. (19), genes encoding for galectin 9, SMAD2 and TGF- $\beta$ were all upregulated at 2 wpi. The genes and pathways described in Alvarez Rojas et al. (19) differed from genes and pathways found in this study at 2 wpi. While SMAD2 was DE in the longitudinal analysis in this study at 16 wpi, it was downregulated. Lack of transcription of interleukin genes was also observed in the previous study (19), while in our study IL16 was downregulated. Since both studies were performed on Merino breed sheep, with the inclusion of control animals, the differences could be attributed to a different strain of $F$. hepatica used, or seasonal differences, if the previous study was conducted in different months. Both Fu et al. (20) and Alvarez Rojas et al. (19) used metacercariae from Baldwin Aquatics, Inc, while in this study Ridgeway metacercariae were used. It is also notable that the DE gene analysis in Alvarez Rojas et al. (19) was performed using NOISeq, which has been shown to produce a comparably large number of false positive results when less than six biological replicates are used (69). Overall, the PBMC studies in sheep show varied results, likely due to breed and infecting strain differences, as well as due to the bioinformatics methods used. It is important to note, to facilitate comparison to previous studies we conducted this study using ovine PBMC. However, future studies to validate these PBMC transcriptomics results could target whole blood collected using PAXgene (Qiagen) or Tempus (Thermo Fisher Scientific) tubes; this approach would immediately preserve whole blood samples and minimise ex vivo variation and RNA degradation (70).

The animals used in our study have also been used to analyse the response to F. hepatica in the HLN at 16 wpi (21). There were common pathways found in PBMC in the infected vs control analysis in this study at 2 wpi and in the HLN at 16 wpi. Specifically, B cell receptor signalling was upregulated in both tissues, and apoptosis and NK cell signalling were downregulated. Crosstalk between DCs and NK cells was downregulated in the hepatic lymph node, while in this study the direction of pathway activation could not be determined, mainly due to a low number of DE genes involved (Supplementary Table S4). In our study, the CD-NK pathway was mainly significant due to the activation of DC receptor genes $C D 69, C D 80$ and $C D 83$. It is important to note that the comparison is made between the acute phase in PBMC and chronic phase in HLN, and therefore the DC-NK cross-talk pathway findings in both studies could be an indication of disease progression from activation of DCs in the acute phase, potentially caused by recognition of parasitic RNAs by TLR9, followed by an inhibition of DC activity in the chronic phase. The involvement of DCs and NK cells in response to F. hepatica is a novel finding in both our study and the study on response to F. hepatica in HLN (21). DCs and NK cells are present in the liver as a part of the resident immune cell population (71). Therefore, the involvement of these cell types in the response of PBMC and lymph node lymphocytes to $F$. hepatica could indicate that these are important cell types responding to the parasite in the liver, and that these cells could be targeted for stimulation of an adaptive immune response.

The aforementioned ovine HLN study used an infected vs control analysis of DE genes, and over $5000 \mathrm{DE}$ genes were detected at 16 wpi (21). However, in our PBMC study only $2 \mathrm{DE}$ genes were found in the infected $v s$ control analysis at 16 wpi. Therefore, a comparison of the chronic response in PBMC and HLN in the present study and the study by Naranjo-Lucena et al. (21) was not possible. In the HLN study, fibrosis was not found in the lymph node tissue of the infected sheep, and the genes TGFB1 and COL1A1 typically associated with liver fibrosis found in the previous ovine studies $(20,72)$ were not DE in the HLN (21). In our PBMC study, these genes were also not DE, even though their expression would be expected to increase due to the "echoing" capacity of PBMC (73). Fibrosis of the liver was not extensive in the sheep from our study, which is confirmed by the lack of significant overexpression of fibrosisrelated genes.

\subsection{Limitations of Longitudinal Analysis}

Larger numbers of DE genes were found in the longitudinal analysis than when the infected and control groups were compared at each time point. However, it is important to note that the $P$ values and fold-changes of these longitudinal DE genes relate to a comparison between the infected animals at week 2 or 16 versus week 0 . The statistical difference between the infected and control group is not accounted for in this case and rather the change in response over time in each group is assessed, and control animals are accounted for by subtracting DE genes present in control animals from the infected animal analysis. This analysis was performed to highlight the potential of developmental or seasonal changes in both groups of sheep, which may lead to a small number of DE genes when infected and control animals are compared. It also highlights the requirement for a suitable control in transcriptomics experiments, where changes in gene expression may be caused by factors other than the infection studied.

The infected $v s$ control analysis identified genes where expression differences were high enough to overcome individual animal differences between the infected and control animal groups. The low number of DE genes found in the main infected $v s$ control analysis also indicates that the systemic response to $F$. hepatica was muted, since larger numbers of DE 
genes were found in the lymph nodes of the same animals at 16 wpi (21).

\subsection{Overall Impact of the Study}

The development of new strategies for the control of fasciolosis in ruminants is urgently required, given the now widespread resistance among liver fluke populations to the only drug effective in killing early immature flukes, and hence protecting against acute fasciolosis in sheep (74). Vaccination is perhaps the most widely-researched and powerful strategy. However, despite decades of focussed research by a number of research groups, significant commercial interest, and limited success in the use of recombinant excretory-secretory proteins from the parasite as vaccine antigens, reviewed by Molina-Hernaández et al. (14) there is as yet no consistent evidence to support the level of vaccine-mediated protection required to bring a vaccine to market. Given the complexity of the target, its complicated life-cycle, lack of immunity from natural infection (56) and propensity to subvert host immune systems (55), these difficulties are in themselves not surprising. In order to overcome the considerable hurdles involved, it may be necessary to adopt novel approaches. Given the difference in disease phenotype between cattle (chronic disease only, economic losses but only very occasional acute disease or mortality) and sheep (acute disease with high morbidity and mortality not unusual, effectively now limiting areas that can be grazed by sheep) (11), one such novel approach might be to ask why the nature of disease varies so much between these two hosts, and whether a "smart vaccine" strategy could, if falling short in terms of rendering sheep resistant to re-infection, could at least limit the pathology to the range of that seen in cattle, thus eliminating mortality and acute morbidity. Exploring the differences in the response of these two species in the acute stage of infection, as we have done in this transcriptomic study, has the potential to indicate strategies, such as use or avoidance of particular adjuvants, and/or co-administration of immunomodulating compounds, that might be useful in this respect.

\subsection{Concluding Remarks}

This is the first study which describes the transcriptomic response of ovine PBMC to F. hepatica with infection duration up to $16 \mathrm{wpi}$ and with control animals included in the experimental design. The study was systematically compared to a similar study in cattle, both of which represent major livestock species that are the targets for development of vaccines as a control measure for fasciolosis. Overall, the infection vs control analysis in our study revealed downregulation of apoptosis and increased cellular metabolism in the acute phase, with the upregulation of the TP53 pathway, and a response to intracellular foreign nucleic acids through TLR9 and RIG-I in the acute phase of infection. The intracellular immune response to RNA molecules could indicate involvement of $F$. hepatica extracellular vesicles in triggering the acute phase response. Based on the most significant DE genes including CD83, expression of TLR9 and a significant DC-NK cross-talk pathway, the involvement of DCs may be key to the acute phase response in sheep. A further longitudinal analysis revealed that an anti-inflammatory response tends to occur in sheep only in the chronic phase of infection, while in cattle the same response is activated as early as $1 \mathrm{wpi}$. This reflects the different phenotype of disease in the two host species, and may point to new control strategies for sheep, such as a potential benefit of using adjuvants in ovine vaccines to trigger an early anti-inflammatory response, or triggering dendritic cell activation. Overall, this study leads to better understanding of host-parasite relationships.

Breed differences and the infecting strain may have affected the DE genes found in this study when compared to other studies. Furthermore, it is apparent that differences in experimental design and bioinformatic approaches used, including selection of controls, use of software packages, or inclusion of batch effects can significantly affect the resulting DE genes. It is recommended that uninfected control animals are used in the experimental design to account for potential seasonal or developmental differences, especially in long term studies. Use of different sheep breeds as well as different $F$. hepatica strains would be desirable in follow-up studies to validate the findings of our study.

\section{DATA AVAILABILITY STATEMENT}

The datasets presented in this study can be found in online repositories. The names of the repository/repositories and accession number(s) can be found below: https://www.ebi.ac. uk/ena/browser/view/PRJEB45790, PRJEB45790.

\section{ETHICS STATEMENT}

The animal study was reviewed and approved by Bioethics Committee of the University of Córdoba (UCO, Spain). Written informed consent was obtained from the owners for the participation of their animals in this study.

\section{AUTHOR CONTRIBUTIONS}

DAN analysed the data and drafted the manuscript. AN-L performed laboratory work. VM-H, AM-M and JP conceived and designed the experimental trial. VM-H collected samples from animals and carried out PBMC isolation. JAB supported laboratory work. DEM supported bioinformatics analysis. GM contributed to the writing of the manuscript. DAN, AN-L, VM-H, JP, GM and DEM contributed to the editing of the manuscript critically for important intellectual content. All authors contributed to the article and approved the submitted version.

\section{FUNDING}

This work was supported by a Science Foundation Ireland grant (14/IA/2304) and a European Union Horizon 2020 programme 
(PARAGONE: vaccines for animal parasites. H2020-EU.3.2. SOCIETAL CHALLENGES, under grant agreement No 635408).

\section{ACKNOWLEDGMENTS}

We would like to thank the staff of the experimental facility from the Universidad de Córdoba for the care of the animals and help provided during the trial.

This article previously appeared as a pre-print on biorXiv, doi: https://doi.org/10.1101/2021.06.21.449266 (75).

\section{SUPPLEMENTARY MATERIAL}

The Supplementary Material for this article can be found online at: https://www.frontiersin.org/articles/10.3389/fimmu.2021. 729217/full\#supplementary-material

Supplementary Figure 1 | Changes in cellular composition in PBMC infected and control groups over time illustrated by cell proportions.

Supplementary Figure 2 | Role of PKR in interferon induction and antiviral response pathway and the genes expressed in PBMC involved in the pathway at 2 wpi.

Supplementary Table S1 | Sample metadata and parameters of sequencing and alignment quality.

\section{REFERENCES}

1. Mas-Coma MS, Esteban JG, Bargues MD. Epidemiology of Human Fascioliasis: A Review and Proposed New Classification. Bull World Health Organ (1999) 77:340-6.

2. WHO. The Selection and Use of Essential Medicines: Report of the WHO Expert Committee, 2005 (Including the 14th Model List of Essential Medicines). Geneva, Switzerland: World Health Organization (2006).

3. Rondelaud D, Dreyfuss G, Bouteille B, Dardé ML. Changes in Human Fasciolosis in a Temperate Area: About Some Observations Over a 28-Year Period in Central France. Parasitol Res (2000) 86:753-7. doi: 10.1007/PL00008563

4. Torgerson P, Claxton J. Epidemiology and Control. in: Fasciolosis Ed E. J. P. Dalton. Wallingford, CABI Publishing (1999) pp 113:149.

5. Bloemhoff Y, Forbes A, Danaher M, Good B, Morgan E, Mulcahy G, et al. Determining the Prevalence and Seasonality of Fasciola Hepatica in PastureBased Dairy Herds in Ireland Using a Bulk Tank Milk ELISA. Irish Vet J (2015) 68:16. doi: 10.1186/s13620-015-0042-5

6. Selemetas N, Ducheyne E, Phelan P, O’Kiely P, Hendrickx G, de Waal T. Spatial Analysis and Risk Mapping of Fasciola Hepatica Infection in Dairy Herds in Ireland. Geospatial Health (2015) 9:281-91. doi: 10.4081/ gh. 2015.350

7. Byrne AW, McBride S, Lahuerta-Marin A, Guelbenzu M, McNair J, Skuce RA, et al. Liver Fluke (Fasciola Hepatica) Infection in Cattle in Northern Ireland: A Large-Scale Epidemiological Investigation Utilising Surveillance Data. Parasites Vectors (2016) 9:209. doi: 10.1186/s13071016-1489-2

8. Quigley A, Sekiya M, Egan S, Wolfe A, Negredo C, Mulcahy, G. Prevalence of Liver Fluke Infection in Irish Horses and Assessment of a Serological Test for Diagnosis of Equine Fasciolosis. Equine Vet J (2017) 49:183-8. doi: 10.1111/ evj. 12577

9. Hurtrez-Boussès S, Meunier C, Durand P, Renaud ,F. Dynamics of HostParasite Interactions: The Example of Population Biology of the Liver Fluke
Supplementary Table S2 | Log2 fold changes and adjusted $P$ values of all differentially expressed genes (Benjamini-Hochberg adjusted $\mathrm{P}$-value $<0.1$ ) in response to F. hepatica at 2 wpi - Infected vs Control analysis. NA - gene not differentially expressed.

Supplementary Table S3 | All significantly overrepresented gene ontology, KEGG and REACTOME pathways in response to $F$. hepatica at 2 wpi. The genes were converted to human orthologs and sorted by adjusted $\mathrm{P}$ value.

Supplementary Table S4 I IPA canonical pathways in PBMC in response to F. hepatica infection in sheep at 2 wpi - infected vc control DE gene analysis. $-\log (p-v a l u e)$ - The probability of association of genes from our dataset with the canonical pathway by random chance alone using Fisher's exact test. ratio - The number of genes in our dataset that meets the cut-off criteria (Padj. $<0.1$ ), divided by the total number of genes involved in that pathway. z-score - Overall activity status of the pathway. $Z<0$ indicates a prediction of an overall decrease in activity while $Z>0$ predicts an overall increase in the activity. NA indicates pathways that are currently ineligible for a prediction.

Supplementary Table S5 | Log2 fold changes and adjusted P values of all differentially expressed genes (Benjamini-Hochberg adjusted $P$ value $<0.05$ ) in response to F. hepatica at 2 and 16 wpi - longitudinal analysis. NA - gene not differentially expressed.

Supplementary Table S6 | Common DE genes in PBMC at the chronic stage of F. hepatica infection in sheep and cattle.

Supplementary Table S7 | IPA canonical pathways in PBMC at acute and chronic stages of F. hepatica infection in sheep and cattle. -log(p-value) - The probability of association of genes from our dataset with the canonical pathway by random chance alone using Fisher's exact test. z-score - Overall activity status of the pathway. $Z<0$ indicates a prediction of an overall decrease in activity while $Z>0$ predicts an overall increase in the activity. NA indicates pathways that are currently ineligible for a prediction.

(Fasciola Hepatica). Microbes Infect (2001) 3:841-9. doi: 10.1016/S1286-4579 (01)01442-3

10. Schweizer G, Braun U, Deplazes P, Torgerson ,PR. Estimating the Financial Losses Due to Bovine Fasciolosis in Switzerland. Vet Rec (2005) 157:188-93. doi: 10.1136/vr.157.7.188

11. Behm C, Sangster ,N. "Fasciolosis". In: J Dalton, editor. Pathology, Pathophysiology and Clinical Aspects. New York: Cabi Publishing, Wallingford Oxon (1999). p. 185-224.

12. Martínez-Valladares M, Cordero-Pérez C, Rojo-Vázquez ,FA. Efficacy of an Anthelmintic Combination in Sheep Infected With Fasciola Hepatica Resistant to Albendazole and Clorsulon. Exp Parasitol (2014) 136:59-62. doi: 10.1016/j.exppara.2013.10.010

13. Kelley JM, Elliott TP, Beddoe T, Anderson G, Skuce P, Spithill ,TW. Current Threat of Triclabendazole Resistance in Fasciola Hepatica. Trends Parasitol (2016) 32:458-69. doi: 10.1016/j.pt.2016.03.002

14. Molina-Hernández V, Mulcahy G, Pérez J, Martínez-Moreno Á, Donnelly S, O'Neill SM, et al. Fasciola Hepatica Vaccine: We may Not be There Yet But We're on the Right Road. Vet parasitol (2015) 208:101-11. doi: 10.1016/ j.vetpar.2015.01.004

15. McManus ,DP. Recent Progress in the Development of Liver Fluke and Blood Fluke Vaccines. Vaccines (2020) 8:553. doi: 10.3390/vaccines 8030553

16. Flynn RJ, Mulcahy G, Elsheikha HM. Coordinating Innate and Adaptive Immunity in Fasciola Hepatica Infection: Implications for Control. Vet Parasitol (2010) 169:235-40. doi: 10.1016/j.vetpar.2010.02.015

17. O’Neill SM, Mills KHG, Dalton JP. Fasciola Hepatica Cathepsin L Cysteine Proteinase Suppresses Bordetella Pertussis-Specific Interferon- $\gamma$ Production In Vivo. Parasite Immunol (2001) 23:541-7. doi: 10.1046/j.1365-3024.2001.00411.x

18. Garcia-Campos A, Correia CN, Naranjo-Lucena A, Garza-Cuartero L, Farries G, Browne JA, et al. Fasciola Hepatica Infection in Cattle: Analyzing Responses of Peripheral Blood Mononuclear Cells (PBMC) Using a Transcriptomics Approach. Front Immunol (2019) 10:2081. doi: 10.3389/ fimmu.2019.02081 
19. Alvarez Rojas CA, Scheerlinck J-P, Ansell BRE, Hall RS, Gasser RB, Jex AR. Time-Course Study of the Transcriptome of Peripheral Blood Mononuclear Cells (PBMCs) From Sheep Infected With Fasciola Hepatica. PLoS One (2016) 11:e0159194. doi: 10.1371/journal.pone.0159194

20. Fu Y, Chryssafidis AL, Browne JA, O'Sullivan J, McGettigan PA, Mulcahy G. Transcriptomic Study on Ovine Immune Responses to Fasciola Hepatica Infection. PloS negl Trop Dis (2016) 10:e0005015. doi: 10.1371/ journal.pntd.0005015

21. Naranjo-Lucena A, Correia CN, Molina-Hernández V, Martínez-Moreno Á, Browne JA, Pérez J, et al. Transcriptomic Analysis of Ovine Hepatic Lymph Node Following Fasciola Hepatica Infection - Inhibition of NK Cell and IgEMediated Signaling. Front Immunol (2021) 12:687579. doi: 10.3389/ fimmu.2021.687579

22. Andrews S. FastQC: A Quality Control Tool for High Throughput Sequence Data (2018). Available at: https://www.bioinformatics.babraham.ac.uk/ projects/fastqc/ (Accessed 20-06-2018 2018).

23. Chen S, Zhou Y, Chen Y, Gu J. Fastp: An Ultra-Fast All-in-One FASTQ Preprocessor. Bioinformatics (2018) 34:i884-90. doi: 10.1093/bioinformatics/ bty560

24. Dobin A, Davis CA, Schlesinger F, Drenkow J, Zaleski C, Jha S, et al. STAR: Ultrafast Universal RNA-Seq Aligner. Bioinformatics (2013) 29:15-21. doi: 10.1093/bioinformatics/bts635

25. Smedley D, Haider S, Ballester B, Holland R, London D, Thorisson G, et al. BioMart-biological Queries Made Easy. BMC Genomics (2009) 10:22. doi: 10.1186/1471-2164-10-22

26. Love MI, Huber W, Anders S. Moderated Estimation of Fold Change and Dispersion for RNA-Seq Data With Deseq2. Genome Biol (2014) 15:550. doi: 10.1186/s13059-014-0550-8

27. Benjamini Y, Hochberg Y. Controlling the False Discovery Rate: A Practical and Powerful Approach to Multiple Testing. J R Stat soc: Ser B (Methodol) (1995) 57:289-300. doi: 10.1111/j.2517-6161.1995.tb02031.x

28. Reimand J, Kull M, Peterson H, Hansen J, Vilo J. G:Profiler-a Web-Based Toolset for Functional Profiling of Gene Lists From Large-Scale Experiments. Nucleic Acids Res (2007) 35:W193-200. doi: 10.1093/nar/gkm226

29. Finotello F, Mayer C, Plattner C, Laschober G, Rieder D, Hackl H, et al. Molecular and Pharmacological Modulators of the Tumor Immune Contexture Revealed by Deconvolution of RNA-Seq Data. Genome Med (2019) 11:34. doi: 10.1186/s13073-019-0638-6

30. Li Z, Ju X, Silveira PA, Abadir E, Hsu W-H, Hart DNJ, et al. CD83: Activation Marker for Antigen Presenting Cells and Its Therapeutic Potential. Front Immunol (2019) 10:1312. doi: 10.3389/fimmu.2019.01312

31. Piedrafita D, Raadsma HW, Prowse R, Spithill TW. Immunology of the HostParasite Relationship in Fasciolosis (Fasciola Hepatica and Fasciola Gigantica). Can J Zool (2004) 82:233-50. doi: 10.1139/z03-216

32. Piedrafita D, Estuningsih E, Pleasance J, Prowse R, Raadsma HW, Meeusen ENT, et al. Peritoneal Lavage Cells of Indonesian Thin-Tail Sheep Mediate Antibody-Dependent Superoxide Radical Cytotoxicity In Vitro Against Newly Excysted Juvenile Fasciola Gigantica But Not Juvenile Fasciola Hepatica. Infect Immun (2007) 75:1954-63. doi: 10.1128/iai.01034-06

33. Toet H, Piedrafita DM, Spithill TW. Liver Fluke Vaccines in Ruminants: Strategies, Progress and Future Opportunities. Int J Parasitol (2014) 44:91527. doi: $10.1016 /$ j.ijpara.2014.07.011

34. Zafra R, Buffoni L, Pérez-Caballero R, Molina-Hernández V, Ruiz-Campillo MT, Pérez J, et al. Efficacy of a Multivalent Vaccine Against Fasciola Hepatica Infection in Sheep. Vet Res (2021) 52:13. doi: 10.1186/s13567-021-00895-0

35. Loo Y-M, Gale MJr. Immune Signaling by RIG-I-Like Receptors. Immunity (2011) 34:680-92. doi: 10.1016/j.immuni.2011.05.003

36. Sánchez-López CM, Trelis M, Jara L, Cantalapiedra F, Marcilla A, Bernal D. Diversity of Extracellular Vesicles From Different Developmental Stages of Fasciola Hepatica. Int J Parasitol (2020) 50:663-9. doi: 10.1016/j.ijpara. 2020.03.011

37. Fromm B, Ovchinnikov V, Høye E, Bernal D, Hackenberg M, Marcilla A. On the Presence and Immunoregulatory Functions of Extracellular Micro RNA s in the Trematode Fasciola Hepatica. Parasite Immunol (2017) 39:e12399. doi: 10.1111/pim.12399

38. Vitour D, Dabo S, Ahmadi Pour M, Vilasco M, Vidalain P-O, Jacob Y, et al. Polo-Like Kinase 1 (PLK1) Regulates Interferon (IFN) Induction by MAVS*. J Biol Chem (2009) 284:21797-809. doi: 10.1074/jbc.M109.018275
39. Kawai T, Akira S. Toll-Like Receptor and RIG-1-Like Receptor Signaling. Ann New York Acad Sci (2008) 1143:1-20. doi: 10.1196/annals.1443.020

40. Rodríguez E, Noya V, Cervi L, Chiribao ML, Brossard N, Chiale C, et al. Glycans From Fasciola Hepatica Modulate the Host Immune Response and TLR-Induced Maturation of Dendritic Cells. PLoS Negl Trop Dis (2016) 9: e0004234. doi: 10.1371/journal.pntd.0004234

41. Falcón C, Carranza F, Martínez FF, Knubel CP, Masih DT, Motrán CC, et al. Excretory-Secretory Products (ESP) From Fasciola Hepatica Induce Tolerogenic Properties in Myeloid Dendritic Cells. Vet Immunol Immunopathol (2010) 137:36-46. doi: 10.1016/j.vetimm.2010.04.007

42. Jenkins T, Brindley P, Gasser R, Cantacessi C. Helminth Microbiomes-a Hidden Treasure Trove? Trends parasitol (2019) 35:13-22. doi: 10.1016/j.pt.2018.10.007

43. McNulty SN, Tort JF, Rinaldi G, Fischer K, Rosa BA, Smircich P, et al. Genomes of Fasciola Hepatica From the Americas Reveal Colonization With Neorickettsia Endobacteria Related to the Agents of Potomac Horse and Human Sennetsu Fevers. PLoS Genet (2017) 13:e1006537. doi: 10.1371/ journal.pgen.1006537

44. Ho PK, Hawkins CJ. Mammalian Initiator Apoptotic Caspases. FEBS J (2005) 272:5436-53. doi: 10.1111/j.1742-4658.2005.04966.x

45. DeLaney AA, Berry CT, Christian DA, Hart A, Bjanes E, Wynosky-Dolfi MA, et al. Caspase-8 Promotes C-Rel-Dependent Inflammatory Cytokine Expression and Resistance Against Toxoplasma Gondii. Proc Natl Acad Sci U S A (2019) 116:11926-35. doi: 10.1073/pnas.1820529116

46. Vousden KH, Prives C. Blinded by the Light: The Growing Complexity of P53. Cell (2009) 137:413-31. doi: 10.1016/j.cell.2009.04.037

47. Kruiswijk F, Labuschagne CF, Vousden KH. P53 in Survival, Death and Metabolic Health: A Lifeguard With a Licence to Kill. Nat Rev Mol Cell Biol (2015) 16:393-405. doi: 10.1038/nrm4007

48. Pellegrino R, Calvisi DF, Ladu S, Ehemann V, Staniscia T, Evert M, et al. Oncogenic and Tumor Suppressive Roles of Polo-Like Kinases in Human Hepatocellular Carcinoma. Hepatology (2010) 51:857-68. doi: 10.1002/hep.23467

49. Kashani A, Holman BWB, Nichols PD, Malau-Aduli AEO. Effect of Dietary Supplementation With Spirulina on the Expressions of AANAT, ADRB3, BTG2 and FASN Genes in the Subcutaneous Adipose and Longissimus Dorsi Muscle Tissues of Purebred and Crossbred Australian Sheep. J Anim Sci Technol (2015) 57:8. doi: 10.1186/s40781-015-0047-3

50. Sarruf DA, Iankova I, Abella A, Assou S, Miard S, Fajas L. Cyclin D3 Promotes Adipogenesis Through Activation of Peroxisome Proliferator-Activated Receptor $\gamma$. Mol Cell Biol (2005) 25:9985-95. doi: 10.1128/mcb.25.22.9985-9995.2005

51. Chen H-H, Wang Y-C, Fann M-J. Identification and Characterization of the CDK12/Cyclin L1 Complex Involved in Alternative Splicing Regulation. Mol Cell Biol (2006) 26:2736-45. doi: 10.1128/mcb.26.7.2736-2745.2006

52. Herrmann A, Fleischer K, Czajkowska H, Müller-Newen G, Becker W. Characterization of Cyclin L1 as an Immobile Component of the Splicing Factor Compartment. FASEB J (2007) 21:3142-52. doi: 10.1096/fj.07-8377com

53. Kang R, Tang D. PKR-Dependent Inflammatory Signals. Sci Signal (2012) 5: pe47. doi: $10.1126 /$ scisignal.2003511

54. Hernandez N, Melki I, Jing H, Habib T, Huang SSY, Danielson J, et al. LifeThreatening Influenza Pneumonitis in a Child With Inherited IRF9 Deficiency. J Exp Med (2018) 215:2567-85. doi: 10.1084/jem.20180628

55. Ryan S, Shiels J, Taggart CC, Dalton JP, Weldon S. Fasciola Hepatica-Derived Molecules as Regulators of the Host Immune Response. Front Immunol (2020) 11:2182. doi: 10.3389/fimmu.2020.02182

56. Clery D, Torgerson P, Mulcahy ,G. Immune Responses of Chronically Infected Adult Cattle to Fasciola Hepatica. Vet parasitol (1996) 62:71-82. doi: 10.1016/0304-4017(95)00858-6

57. Bossaert K, Jacquinet E, Saunders J, Farnir F, Losson ,B. Cell-Mediated Immune Response in Calves to Single-Dose, Trickle, and Challenge Infections With Fasciola Hepatica. Vet parasitol (2000) 88:17-34. doi: 10.1016/S0304-4017(99)00200-9

58. Kawasaki T, Kawai T. Toll-Like Receptor Signaling Pathways. Front Immunol (2014) 5:461. doi: 10.3389/fimmu.2014.00461

59. Cutroneo KR. TGF- $\beta$-Induced Fibrosis and SMAD Signaling: Oligo Decoys as Natural Therapeutics for Inhibition of Tissue Fibrosis and Scarring. Wound Repair Regener (2007) 15:S54-60. doi: 10.1111/j.1524-475X.2007.00226.x

60. Haçariz O, Sayers G, Flynn RJ, Lejeune A, Mulcahy G. IL-10 and TGF- $\beta 1$ are Associated With Variations in Fluke Burdens Following Experimental Fasciolosis in Sheep. Parasite Immunol (2009) 31:613-22. doi: 10.1111/ j.1365-3024.2009.01135.x 
61. Altamirano-Barrera A, Barranco-Fragoso B, Méndez-Sánchez N. Management Strategies for Liver Fibrosis. Ann Hepatol (2017) 16:48-56. doi: 10.5604/16652681.1226814

62. Tiggelman AMBC, Boers W, Linthorst C, Sala M, Chamuleau RAEM. Collagen Synthesis by Human Liver (Myo)Fibroblasts in Culture: Evidence Fora Regulatory Role of IL-1 $\beta, I L-4$, Tgf $\beta$ and IFN Gamma. J Hepatol (1995) 23:307-17. doi: 10.1016/S0168-8278(95)80010-7

63. McCusker P, McVeigh P, Rathinasamy V, Toet H, McCammick E, O'Connor A, et al. Stimulating Neoblast-Like Cell Proliferation in Juvenile Fasciola Hepatica Supports Growth and Progression Towards the Adult Phenotype In Vitro. PLoS Negl Trop Dis (2016) 10:e0004994. doi: 10.1371/journal.pntd.0004994

64. Ruiz-Campillo MT, Hernandez VM, Escamilla A, Stevenson M, Perez J, Martinez-Moreno A, et al. Immune Signatures of Pathogenesis in the Peritoneal Compartment During Early Infection of Sheep With Fasciola Hepatica. Sci Rep (2017) 7:1-14. doi: 10.1038/s41598-017-03094-0

65. Valero MA, Bargues MD, Khoubbane M, Artigas P, Quesada C, Berinde L, et al. Higher Physiopathogenicity by Fasciola Gigantica Than by the Genetically Close F. Hepatica: Experimental Long-Term Follow-Up of Biochemical Markers. Trans R Soc Trop Med Hyg (2016) 110:55-66. doi: 10.1093/trstmh/trv110

66. Liu N, Li H, Liu K, Yu J, Cheng M, De W, et al. Differential Expression of Genes and Proteins Associated With Wool Follicle Cycling. Mol Biol Rep (2014) 41:5343-9. doi: 10.1007/s11033-014-3405-1

67. Hansen D, Clery D, Estuningsih S, Widjajanti S, Partoutomo S, Spithill TW. Immune Responses in Indonesian Thin Tail and Merino Sheep During a Primary Infection With Fasciola Gigantica: Lack of a Specific IgG2 Antibody Response is Associated With Increased Resistance to Infection in Indonesian Sheep. Int J parasitol (1999) 29:1027-35. doi: 10.1016/S0020-7519(99)00038-7

68. Pleasance J, Wiedosari E, Raadsma H, Meeusen E, Piedrafita ,D. Resistance to Liver Fluke Infection in the Natural Sheep Host is Correlated With a Type-1 Cytokine Response. Parasite Immunol (2011) 33:495-505. doi: 10.1111/ j.1365-3024.2011.01305.x

69. Schurch NJ, Schofield P, Gierliński M, Cole C, Sherstnev A, Singh V, et al. How Many Biological Replicates are Needed in an RNA-Seq Experiment and Which Differential Expression Tool Should You Use? RNA (2016) 22:839-51. doi: 10.1261/rna.053959.115

70. Härtel C, Bein G, Müller-Steinhardt M, Klüter H. Ex Vivo Induction of Cytokine mRNA Expression in Human Blood Samples. J Immunol Methods (2001) 249:63-71. doi: 10.1016/s0022-1759(00)00334-3
71. Robinson MW, Harmon C, O’Farrelly ,C. Liver Immunology and its Role in Inflammation and Homeostasis. Cell Mol Immunol (2016) 13:267-76. doi: $10.1038 / \mathrm{cmi} .2016 .3$

72. Alvarez Rojas CA, Ansell BRE, Hall RS, Gasser RB, Young ND, Jex AR, et al. Transcriptional Analysis Identifies Key Genes Involved in Metabolism, Fibrosis/Tissue Repair and the Immune Response Against Fasciola Hepatica in Sheep Liver. Parasites Vectors (2015) 8:124. doi: 10.1186/ s13071-015-0715-7

73. Chaussabel D, Pascual V, Banchereau ,J. Assessing the Human Immune System Through Blood Transcriptomics. BMC Biol (2010) 8:84. doi: 10.1186/ 1741-7007-8-84

74. Castro-Hermida JA, González-Warleta M, Martínez-Sernández V, Ubeira FM, Mezo M. Current Challenges for Fasciolicide Treatment in Ruminan Livestock. Trends Parasitol (2021) 37:430-44. doi: 10.1016/j.pt.2020.12.003

75. Niedziela DA, Naranjo-Lucena A, Molina-Hernández V, Browne JA, Martínez-Moreno A, Pérez J, et al. Timing of Transcriptomic Peripheral Blood Mononuclear Cell Responses of Sheep to Fasciola hepatica Infection Differs From Those of Cattle, Reflecting Different Disease Phenotypes. biorXiv (2021). doi: 10.1101/2021.06.21.449266

Conflict of Interest: The authors declare that the research was conducted in the absence of any commercial or financial relationships that could be construed as a potential conflict of interest.

Publisher's Note: All claims expressed in this article are solely those of the authors and do not necessarily represent those of their affiliated organizations, or those of the publisher, the editors and the reviewers. Any product that may be evaluated in this article, or claim that may be made by its manufacturer, is not guaranteed or endorsed by the publisher.

Copyright (c) 2021 Niedziela, Naranjo-Lucena, Molina-Hernández, Browne, Martinez-Moreno, Pérez, MacHugh and Mulcahy. This is an open-access article distributed under the terms of the Creative Commons Attribution License (CC BY). The use, distribution or reproduction in other forums is permitted, provided the original author(s) and the copyright owner(s) are credited and that the original publication in this journal is cited, in accordance with accepted academic practice. No use, distribution or reproduction is permitted which does not comply with these terms. 